\title{
Synthesis of nanocomposites using glasses and mica as templates
}

\author{
D CHAKRAVORTY*, S BASU, B N PAL ${ }^{\dagger}$, P K MUKHERJEE ${ }^{\dagger \dagger}$, B GHOSH, \\ K CHATTERJEE ${ }^{\#}$, A BOSE, $S$ BHATTACHARYA and A BANERJEE ${ }^{\S}$ \\ DST Unit on Nano Science and MLS Professor's Unit, Indian Association for the Cultivation of Science, \\ Kolkata 700 032, India \\ ${ }^{\dagger}$ Department of Materials Science and Engineering, Johns Hopkins University, Maryland, USA \\ ${ }^{\dagger \dagger}$ Department of Physics, Durgapur Government College, Durgapur 713214 , India \\ \#Department of Physics, Vidyasagar University, Midnapore 721 102, India \\ ${ }^{\S}$ Chemistry Division, Indian Institute of Chemical Biology, Kolkata 700 032, India
}

\begin{abstract}
Various nanocomposites were synthesized using either a silica-based glass or mica crystallites as the medium. In some cases by an oxidation or a sulfidation treatment a core-shell nanostructure could be generated. Iron-iron oxide core-shell structured nanocomposites exhibited excellent humidity sensing behaviour. Gold-gold sulfide core-shell nanorods exhibited a number of optical absorption peaks which arose because of their structural characteristics. Nanoparticles of silver and silver oxide could be aligned in a polymethylmethacrylate film by an a.c. electric field of $1 \mathrm{MHz}$ frequency. The composites showed large sensitivity to relative humidity. Lead sulfide nanowires of diameter, $1.2 \mathrm{~nm}$, were grown within the nanochannels of Na-4 mica. These exhibited a semiconductor to metal transition at around $300 \mathrm{~K}$. This arose because of high pressure generated on the nanowires. Copper sulfide nanowires grown within the Na-4 mica channels showed metallic behaviour. Silver core-silver orthosilicate shell nanostructures developed within a silicate glass medium showed discontinuous changes in resistivity at some specific temperatures. This was explained as arising due to excitation of Lamb modes at certain pressures generated because of thermal expansion mismatch of the core and the shell phases. Optical properties of iron core-iron oxide shell nanocomposites when analysed by effective medium theory led to the result of a metal non-metal transition for particle diameters below a critical value. Similar results were obtained from optical absorption data of silver nanoparticles grown in a tetrapeptide solution.
\end{abstract}

Keywords. Synthesis; nanocomposites; glasses; mica.

\section{Introduction}

Nanocomposites are materials in which a phase with nanoscale dimensions is dispersed within a matrix. A wide variety of nanocomposites have been studied in recent years (Watt et al 2005; Ng et al 2006; Dutta et al 2006; Park and Park 2006; Kesanli et al 2006). These systems have acquired significance because of interesting physical properties exhibited by them. Various physical and chemical techniques have been used to synthesize these materials. We have synthesized a number of nanocomposites using either a silica-based glass or a mica structure as the medium. In most cases the nanosized phase consisted of a metal. By suitable oxidation of the composite a core-shell nanostructure was also developed in them. The materials exhibited interesting electrical and optical properties. In some cases, by a suitable choice of the core and the shell phase high pressure could be generated in the nanoscale regions of the composites. This gave rise to unusual behaviour. In this paper, we review the systems studied so far and delineate the properties measured.

*Author for correspondence (mlsdc@iacs.res.in)

\section{Experimental}

\subsection{Nanocomposites with iron-iron oxide core-shell structure}

The composites were prepared by first growing iron nanoparticles within a silica based gel by using an electrodeposition technique (Banerjee et al 1999). A shell of $\mathrm{Fe}_{3} \mathrm{O}_{4}$ was then formed around the iron nanoparticles by subjecting the composite to an oxidation treatment.

The composition of the gel to be prepared was chosen as $40 \mathrm{Fe}\left(\mathrm{NO}_{3}\right)_{3}$ and $60 \mathrm{SiO}_{2}$. The precursors used were $\mathrm{Fe}$ $\left(\mathrm{NO}_{3}\right)_{3} \cdot 9 \mathrm{H}_{2} \mathrm{O}$ and $\mathrm{Si}\left(\mathrm{OC}_{2} \mathrm{H}_{5}\right)_{4}$, respectively. A solution was prepared by dissolving the required amount of $\mathrm{Fe}\left(\mathrm{NO}_{3}\right)_{3}$ in distilled water and ethyl alcohol. The solution was stirred by a magnetic stirrer. A second solution was made by dissolving a calculated amount of $\mathrm{Si}\left(\mathrm{OC}_{2} \mathrm{H}_{5}\right)_{4}$ in distilled water and ethyl alcohol. The above solutions were mixed and stirred for $1 \mathrm{~h}$ and the $\mathrm{pH}$ value was maintained at 4 . Cleaned microscope slides of dimension $2 \mathrm{~cm} \times 2 \mathrm{~cm} \times$ $1 \mathrm{~mm}$ were dipped into the sol and drawn at a speed of $1 \mathrm{~mm} / \mathrm{s}$. The slides were dried under ordinary atmosphere for a few hours. Films of thickness around $4 \mu \mathrm{m}$ were ob- 
tained which were transparent. Two silver electrodes (Acheson Colloiden B.V. Holland) were painted on the film surface at a separation of $2 \mathrm{~mm}$. A d.c. voltage of $5 \mathrm{~V}$ was applied between the electrodes for around $30 \mathrm{~s}$ at room temperature. The formation of metallic filaments was indicated by a sudden increase of current to a value of $\sim 1 \mathrm{~mA}$. The specimens were then subjected to an oxidation treatment by heating them to a temperature of $400 \mathrm{~K}$ for a period varying from $1-4 \mathrm{~h}$.

The resultant structure was studied by a Leitz Laborlux 18 Pol S optical microscope in the transmission mode. The microstructure of the electrodeposited nanocomposites was investigated by a JEOL 2010 transmission electron microscope.

D.C. electrical resistances of different specimens were measured by a Keithley 617 Electrometer.

Humidity sensing behaviour of these nanocomposites was studied by subjecting them to nitrogen gas flow whose moisture content could be varied by passing it through either a $\mathrm{P}_{2} \mathrm{O}_{5}$ guard tube or a water container. The relative humidity $(\mathrm{RH})$ value was measured by a Barigo Hygrometer (made in Germany).

\subsection{Gold-gold sulfide core-shell structured nanorods}

Gold nanorods were, first of all, grown by an electrodeposition technique using polycarbonate membranes as templates (Chatterjee et al 2006). The membranes with pore diameter, $100 \mathrm{~nm}$ and thickness, $1 \mu \mathrm{m}$ (supplied by Whatman, Nuclepore, Clifton, NJ, USA) were painted on one surface by a silver paste (supplied by Acheson Colloiden B. V. Holland, The Netherlands) which acted as the cathode. The experimental arrangement used is shown in figure 6 . Tetrachloroauric acid $\left(\mathrm{HAuCl}_{4} \cdot x \mathrm{H}_{2} \mathrm{O}\right)$ was used as the electrolyte and a gold plate used as the anode.

A d.c. voltage of $1.5 \mathrm{~V}$ was applied to the electrolytic cell for $5 \mathrm{~min}$. In the process gold nanorods were formed. The polycarbonate film containing gold nanorods was dissolved in chloroform. The resulting solution was mixed with $2 \mathrm{mg}$ thiourea [ $\left.\left(\mathrm{NH}_{2}\right)_{2} \mathrm{CS}\right]$. A few drops of ammoniacal solution were added to the former and it was subjected to a heat treatment at $343 \mathrm{~K}$. As a result the gold nanorods were coated with $\mathrm{Au}_{2} \mathrm{~S}$. The microstructure of samples both prior to and after the sulfidation treatment were investigated by a JEOL 2010 transmission electron microscope.

For optical property measurements, $\mathrm{Au}_{2} \mathrm{~S}$ coated gold nanorods dispersed in chloroform were mounted in the specimen chamber of a UV-2101 PC ultraviolet-visible scanning spectrophotometer (Japan). The absorption spectra were recorded by keeping a chloroform containing dissolved polycarbonate film at the reference beam position.

\subsection{Aligned binary system of nanoparticles in a polymer film}

The approach was to first prepare nanoparticles of $\mathrm{Ag}_{2} \mathrm{O}$ and $\mathrm{Ag}$, respectively and then a solution containing these particles was subjected to an a.c. electric field.

A solution was prepared by dissolving $1 \mathrm{~g}$ of analytical grade silver nitrate in 40 c.c. distilled water. A second solution was made by dissolving $0.117 \mathrm{~g}$ of $\mathrm{NaOH}$ pellet in 20 c.c. distilled water. The $\mathrm{pH}$ of the latter solution was $\sim 10$. One drop of $\mathrm{NaOH}$ solution was added to the first solution and the mixture was stirred vigorously for a few minutes. This precipitated $\mathrm{Ag}_{2} \mathrm{O}$. Ultrafine particles of $\mathrm{Ag}_{2} \mathrm{O}$ were obtained by filtering the solution through a Whatman filter paper. A reasonable amount of $\mathrm{Ag}_{2} \mathrm{O}$ was extracted by repeating the process several times on the filtrate. After drying the precipitate in vacuum, the $\mathrm{Ag}_{2} \mathrm{O}$ particles were heated at $540 \mathrm{~K}$ for $1 \mathrm{~h}$. Due to dissociation of $\mathrm{Ag}_{2} \mathrm{O}$ to $\mathrm{Ag}$ and $\mathrm{O}_{2}$ at temperatures above $500 \mathrm{~K}$ ultrafine silver particles were obtained by the above heat treatment. Particle sizes of the powders (both $\mathrm{Ag}_{2} \mathrm{O}$ and $\mathrm{Ag}$ ) were characterized by a JEM 2010 transmission electron microscope. Next a polymethylmethacrylate (PMMA) solution was made in acetonitrile and a mixture (weighing $4 \mathrm{mg}$ ) containing equal amounts of $\mathrm{Ag}$ and $\mathrm{Ag}_{2} \mathrm{O}$ was poured into the same. The solution was subjected to sonication. Two silver paint electrodes obtained from B. V. Colloiden, Netherlands, were applied on a glass slide at a separation of $1 \mathrm{~mm}$ and a few drops of the polymer solution containing $\mathrm{Ag}$ and $\mathrm{Ag}_{2} \mathrm{O}$ nanoparticles were placed in between. A.C. electric field at frequencies in the range $200 \mathrm{~Hz}$ to $1 \mathrm{MHz}$ was applied across the electrodes. Acetonitrile evaporated within $30 \mathrm{~s}$ and a stable polymer film formed with specific patterns of the binary nanoparticles.

A Hitachi S-415A Scanning Electron Microscope was used to study the microstructure. D.C. electrical resistances were measured over the temperature range 270 $630 \mathrm{~K}$ by a Keithley 617 electrometer.

\subsection{Lead sulfide nanowires grown in mica channels}

The channels within a high-charge density sodium fluorophlogopite mica channels of chemical composition, $\mathrm{Na}_{4} \mathrm{Mg}_{6} \mathrm{Al}_{4} \mathrm{Si}_{4} \mathrm{O}_{26} \mathrm{~F}_{4} \cdot x \mathrm{H}_{2} \mathrm{O}$ (Kodama and Komarneni 1999), were used to grow $\mathrm{PbS}$ nanowires of diameter, $\sim 1.2 \mathrm{~nm}$.

Mica of the above mentioned composition is commonly referred to as $\mathrm{Na}-4$ mica. This was prepared by a sol-gel method. The chemicals used were $\mathrm{Al}\left(\mathrm{NO}_{3}\right) \cdot 9 \mathrm{H}_{2} \mathrm{O}$, $\mathrm{Mg}\left(\mathrm{NO}_{3}\right)_{2} \cdot 6 \mathrm{H}_{2} \mathrm{O}$ and $\mathrm{Si}\left(\mathrm{OC}_{2} \mathrm{H}_{5}\right)_{4}$. The latter were dissolved in $\mathrm{C}_{2} \mathrm{H}_{5} \mathrm{OH}$ and the solution was stirred for $3 \mathrm{~h}$. The amounts of the compounds were chosen such that the final composition would be $\mathrm{MgO} \cdot \mathrm{Al}_{2} \mathrm{O}_{3} \cdot 2 \mathrm{SiO}_{2}$. The gel was dried at $373 \mathrm{~K}$ and the calcination done at $748 \mathrm{~K}$ for $12 \mathrm{~h}$. The gel powder was mixed thoroughly with an equal amount of $\mathrm{NaF}$ powder. The mixture was subjected 
to a heat treatment at $1163 \mathrm{~K}$ for $18 \mathrm{~h}$ in a platinum crucible. After washing the reaction products with deionized water and then with saturated boric acid they were washed with $1 \mathrm{M} \mathrm{NaCl}$ to saturate all exchange sites with $\mathrm{Na}^{+}$. After subsequent washing with deionized water the solid was dried at $333 \mathrm{~K}$ for 3 days. $2 \mathrm{~g}$ of Na-4 mica powder was then immersed into $25 \mathrm{cc}$ of $0.4(\mathrm{M}) \mathrm{Pb}\left(\mathrm{NO}_{3}\right)_{2}$ to which $0.5 \mathrm{M} \mathrm{NaCl}$ solution was added. This brought about an ion exchange reaction of the type $\mathrm{Pb}^{2+} \leftrightarrow 2 \mathrm{Na}^{+}$which continued for a period of 4 weeks at $303 \mathrm{~K}$. After separating the ion exchanged powder from the solution by a centrifuge, it was subjected to a heat treatment in $\mathrm{H}_{2} \mathrm{~S}$ at $673 \mathrm{~K}$ for $1 \mathrm{~h}$. This produced $\mathrm{PbS}$ wires within the $\mathrm{Na}-4$ mica channels.

The microstructure of the samples was investigated by a JEOL Model JEM 2010 transmission electron microscope.

Electrical measurements were carried out on cold compacted specimens using a pressure of 20 tons $/ \mathrm{cm}^{2}-$ the specimens having a diameter, $1 \mathrm{~cm}$ and thickness, $1 \mathrm{~mm}$. Before d.c. measurement, the specimens were sintered at $973 \mathrm{~K}$ for $1 \mathrm{~h}$ in a nitrogen atmosphere. Silver paste supplied by B. V. Colloiden, The Netherlands, was used as electrodes. D.C. resistance at different temperatures were measured by Keithley 617 Electrometer. Dielectric properties were measured by a Hewlett Packard Impedance Analyser (HP4192A).

\subsection{Copper sulfide nanowires grown within $\mathrm{Na}-4$ nanochannels}

Na-4 mica channels were exploited to grow wires of copper sulfide.

$\mathrm{Na}-4$ mica was first prepared by following the procedure described in the previous section. $2 \mathrm{~g}$ of the gel powder was immersed in a solution consisting of $0.4 \mathrm{M}$ $\mathrm{CuCl}_{2} \cdot 2 \mathrm{H}_{2} \mathrm{O}$ and $0.5 \mathrm{M} \mathrm{NaCl}$. This brought about an ion exchange reaction $\mathrm{Cu}^{2+} \leftrightarrow 2 \mathrm{Na}^{+}$. The reaction was allowed to take place over a period of 4 weeks at $303 \mathrm{~K}$. After separating the ion exchanged powder from the solution by a centrifuge the former was subjected to heat treatment at $673 \mathrm{~K}$ for $1 \mathrm{~h}$ in $\mathrm{H}_{2} \mathrm{~S}$ atmosphere. This led to the formation of copper sulfide nanowires within the crystal channels of Na-4 mica.

The procedures for microstructural studies, d.c. resistance and dielectric measurements were identical to those described in the previous section.

2.6 Nanocomposites with silver core-silver orthosilicate shell structures

A silicate glass with suitable composition was chosen to first of all grow nanoparticles of metallic silver which were interconnected. After suitable heat treatment a crystalline phase viz. silver orthosilicate, was grown around the silver nuclei. With this system it was possible to study the effect of high pressure on the ionic conductivity of the nano-shell comprising of silver orthosilicate (Ghosh et al 2004).

Glasses of compositions, $15 \mathrm{Na}_{2} \mathrm{O}, 15 \mathrm{Li}_{2} \mathrm{O}, 12 \mathrm{CaO}$, $3 \mathrm{Al}_{2} \mathrm{O}_{3}, 55 \mathrm{SiO}_{2}$, were prepared by melting a mixture of appropriate chemicals and then pouring it on a brass mould at room temperature. Glass particles of size, $\sim 5 \mu \mathrm{m}$, were obtained after grinding the quenched glass pieces. The former were immersed in a molten bath of silver nitrate at $583 \mathrm{~K}$ for $6 \mathrm{~h}$ to bring about ion exchange of the type $\mathrm{Li} \leftrightarrow \mathrm{Ag}^{+}$and $\mathrm{Na}^{+} \leftrightarrow \mathrm{Ag}^{+}$, respectively. The treated powder was reduced in hydrogen at $673 \mathrm{~K}$ for $30 \mathrm{~min}$. Silver particles were formed in the glass by this step. Electrical measurement carried out on a cold compacted pellet of the reduced powder showed metallic conduction confirming percolation of the silver nanoparticles. The glass powder prepared as above was subjected to a two-stage heat treatment, viz. at $833 \mathrm{~K}$ and $993 \mathrm{~K}$, respectively for different periods to nucleate and grow the silver orthosilicate phase.

The microstructural and electrical studies were carried out by methods delineated in previous sections.

\subsection{Nanocomposites with iron-iron oxide core-shell structures}

Iron nanoparticles were first grown within a gel-derived silicate glass and then by oxidation treatment an iron oxide shell was formed (Basu and Chakravorty 2006).

The target gel composition was $55 \mathrm{Fe}_{2} \mathrm{O}_{3} \cdot 45 \mathrm{SiO}_{2}$. The precursor chemicals were $\mathrm{FeCl}_{3}$ and $\mathrm{Si}\left(\mathrm{OC}_{2} \mathrm{H}_{5}\right)_{4}$. The sol was prepared by mixing weighed amounts of chemicals in ethyl alcohol, distilled water and $\mathrm{Si}\left(\mathrm{OC}_{2} \mathrm{H}_{5}\right)_{4} .1 \mathrm{cc}$ of $\mathrm{HCl}$ was added. This was allowed to gel for a period of two weeks. The gel was subjected to a reduction treatment in hydrogen at $923 \mathrm{~K}$ for $30 \mathrm{~min}$. The powders were then heated in ordinary atmosphere at temperatures in the range $573-973 \mathrm{~K}$ for $30 \mathrm{~min}$.

The microstructural studies were carried out by a JEOL2010 transmission electron microscope. Optical absorption spectra were measured by a UV-2101 PC UVVIS scanning spectrophotometer of Shimadzu-make after dispersing the nanocomposite powders in ethyl alcohol in a test tube.

\subsection{Silver nanoparticles grown in an oligopeptide}

A water-soluble tetrapeptide solution was used for making silver nanoparticles (Bhattacharya et al 2006).

The precursors used were silver nitrate, tetrapeptide and methanol. Preparation of tetrapeptide is described elsewhere (Bodanszky and Bodanszky 1984). Two solutions were prepared, one of tetrapeptide in methanol and water and the other of silver nitrate in methanol and water. 
The first solution was then added to the second one under stirring in a dark chamber. The $\mathrm{pH}$ of the solution was adjusted to $\sim 8$ by suitable addition of $\mathrm{NaOH}$ solution. Colloidal silver particles were produced due to oxidation of the tyrosine residues of the tetrapeptide.

The microstructural and optical absorption studies were carried out by the procedure described in previous sections.

\section{Results and discussion}

\subsection{Iron-iron oxide core-shell structure}

In figure 1 is shown an optical micrograph of a gel sample in which iron metal was grown with a fractal structure by application of a d.c. voltage of $5 \mathrm{~V}$ and subsequent oxidation treatment was given at $400 \mathrm{~K}$ for $2 \mathrm{~h}$. The fractal dimension was calculated to be 1.82 (Banerjee et al 1999). This has been ascribed to the generalized-diffusionlimited-aggregation model (Matshushita et al 1986).

Figure 2 shows the transmission electron micrograph for the specimen above. It is evident that the fractal structure is comprised of nanosized particles. From the distribution of particle sizes, a median diameter of $10 \mathrm{~nm}$ was extracted. Figure 3 shows the core-shell nanostructure of the particles. From the electron diffraction pattern of these particles the interplanar spacings were calculated. The latter confirmed that the core and the shell were made up of $\mathrm{Fe}$ and $\mathrm{Fe}_{3} \mathrm{O}_{4}$, respectively (Pal et al 2005a).

Figure 4 gives the variation of surface resistivity of a specimen (subjected to a $2 \mathrm{~h}$ oxidation treatment) as a

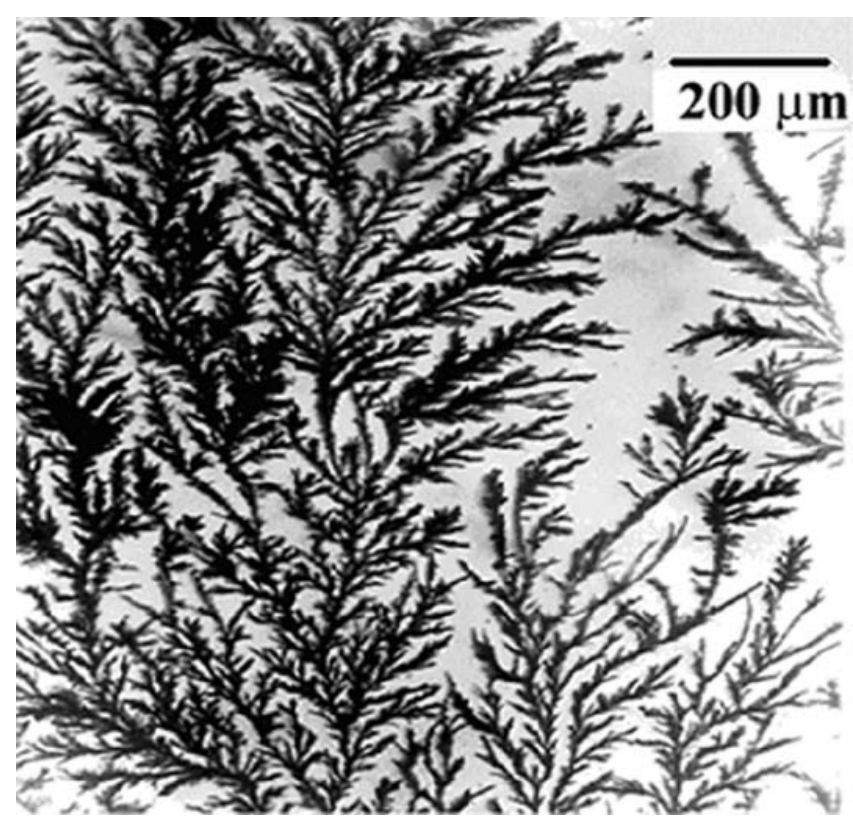

Figure 1. Optical micrograph of a gel-derived $\mathrm{Fe}\left(\mathrm{NO}_{3}\right)_{3}-\mathrm{SiO}_{2}$ film subjected to a d.c. voltage of $5 \mathrm{~V}$ and subsequently heated at $400 \mathrm{~K}$ for $2 \mathrm{~h}$ function of inverse temperature. The electrical conduction evidently is controlled by the $\mathrm{Fe}_{3} \mathrm{O}_{4}$ shell. The latter is known to contain both $\mathrm{Fe}^{2+}$ and $\mathrm{Fe}^{3+}$ ions. Hence a small polaron conduction model (Mott 1968) was found to be operative in this system. In this model resistivity, $\rho$, is given by

$$
\rho=\frac{k T R}{v_{0} e^{2} c(1-c)} \exp (2 \alpha R) \exp \left(\frac{W}{k T}\right),
$$

where $k$ is the Boltzmann constant, $T$ the temperature, $R$ the intersite separation, $v_{0}$ the optical phonon frequency,

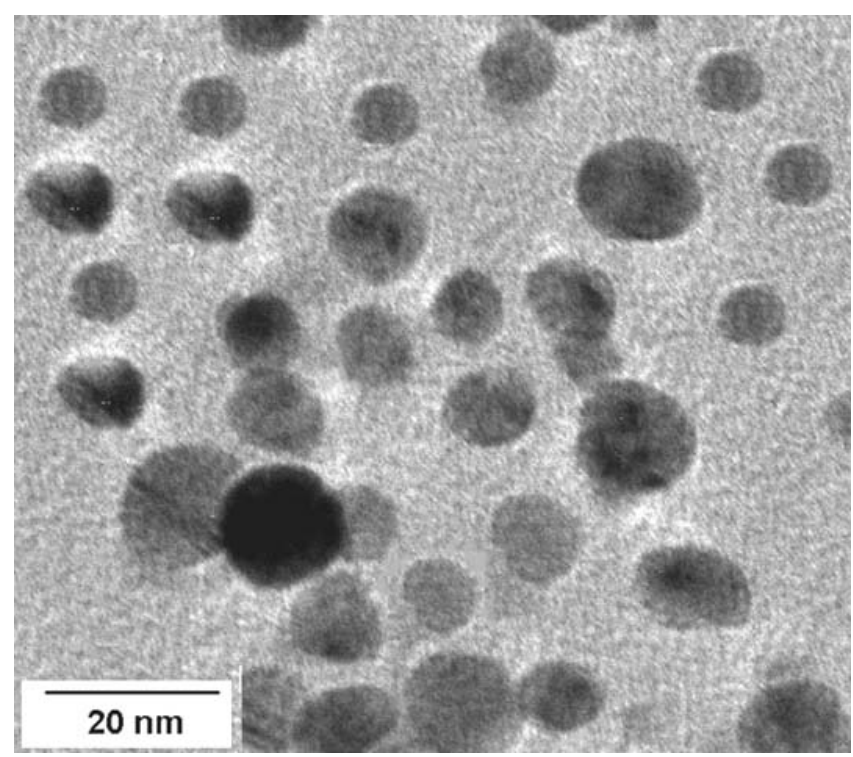

Figure 2. Transmission electron micrograph for specimen in figure 1.

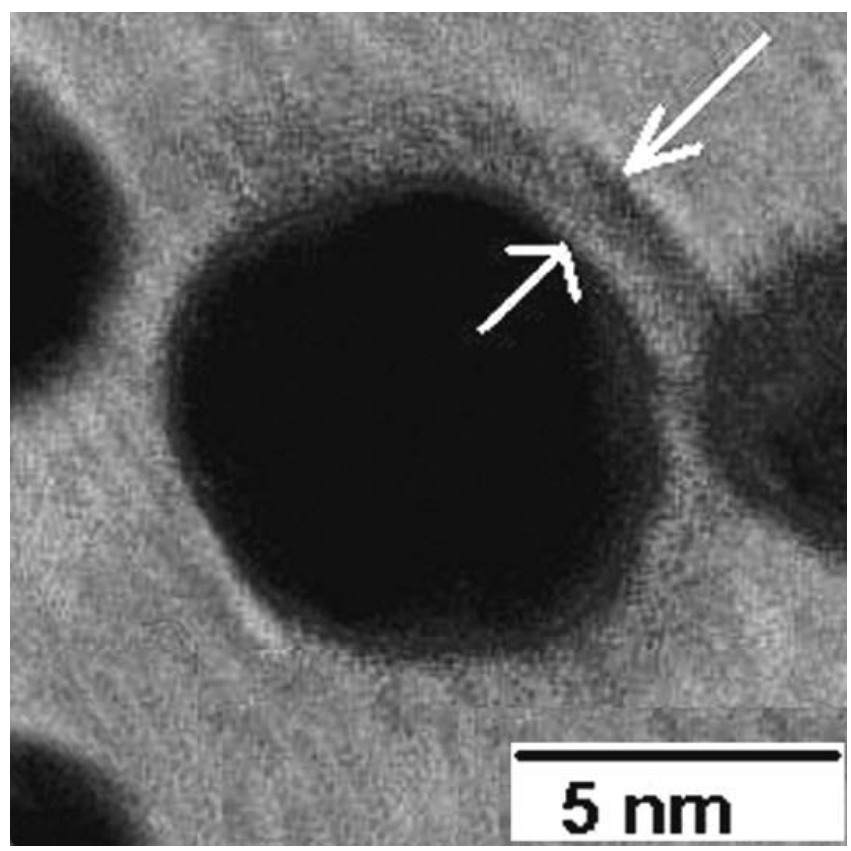

Figure 3. Transmission electron micrograph of the core-shell nanostructure. 
$e$ the electronic charge, $c$ the ratio of $\left[\mathrm{Fe}^{2+}\right] /[\mathrm{Fe}]_{\text {total }}, \alpha^{-1}$ the localization length of the localized state at the $\mathrm{Fe}$ ion site and $W$ the activation energy for hopping condition. The experimental data were least square fitted to (1) and the extracted parameters are $W=0.66 \mathrm{eV}, \alpha=0.9 \AA^{-1}$, $R=2.9 \AA, c=0.9$ and $v_{0}=0.1 \times 10^{13} \mathrm{~s}^{-1}$. These are typi-

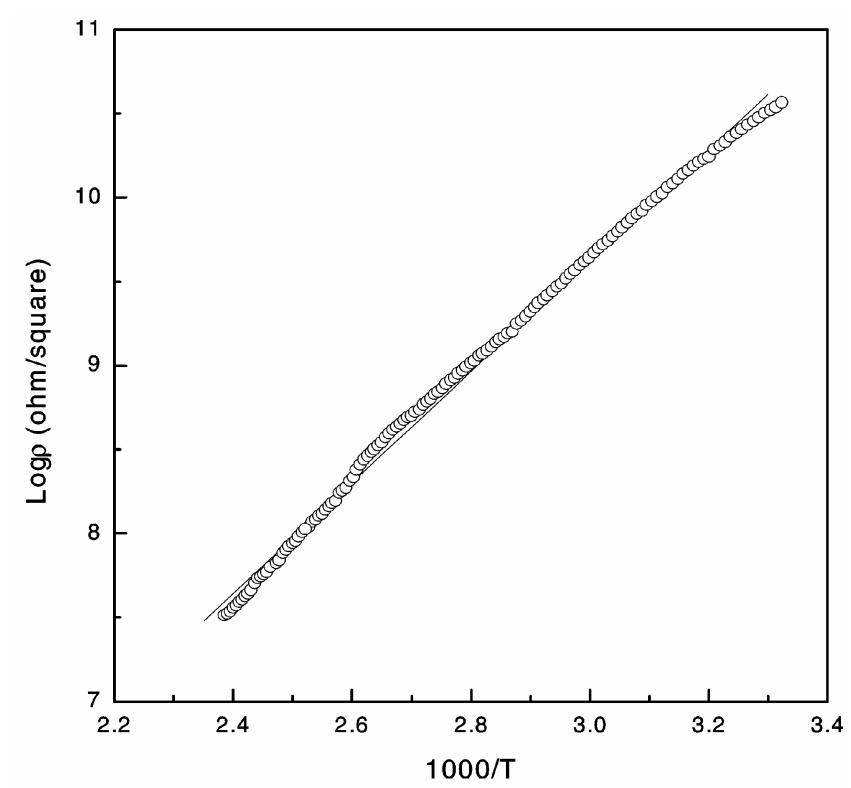

Figure 4. Variation of surface resistivity as a function of inverse temperature for the nanocomposite subjected to $2 \mathrm{~h}$ oxidation treatment.

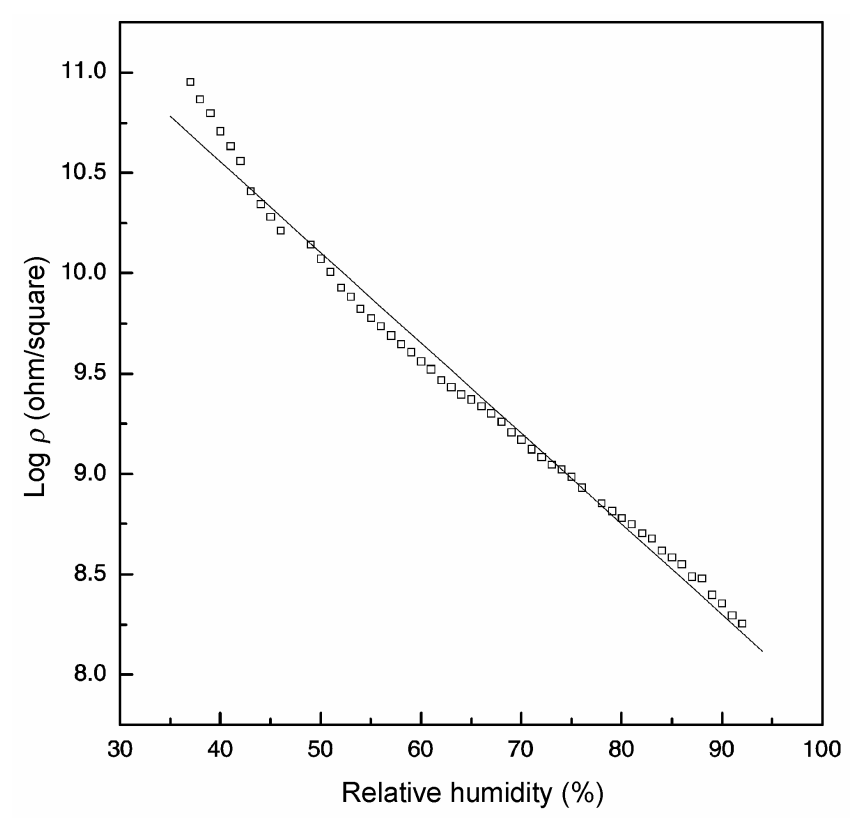

Figure 5. Surface resistivity variation as a function of relative humidity for specimen subjected to $2 \mathrm{~h}$ oxidation treatment. cal for other samples and are consistent with the model used.

Figure 5 depicts the variation of the surface resistivity as a function of relative humidity in the case of specimen subjected to a $2 \mathrm{~h}$ oxidation treatment. This is typical of the other specimens. An almost three-order of magnitude resistivity change can be seen for the variation of relative humidity from 30 to $90 \%$. The electrical conduction under humid conditions was found to be electronic in origin. This is believed to arise due to electron injection into the $\mathrm{Fe}_{3} \mathrm{O}_{4}$ phase by water molecules chemisorbed on $\mathrm{Fe}_{3} \mathrm{O}_{4}$ shells.

\subsection{Gold-gold sulfide nanorods}

Figure 7(a) is an electron micrograph for a $\mathrm{Au}_{2} \mathrm{~S}$ coated gold nanorod. Figure 7(b) is an electron diffraction pattern obtained from figure 7 (a). The interplanar spacing, $d_{h k l}$ values, were calculated from the measured diameters of the diffraction rings. In table 1 , the experimentally determined $d_{h k l}$ values have been compared with standard ASTM data for gold and gold sulfide phases, respectively. It is evident that both these phases are present in the samples prepared.

Figure 8 shows typical extinction coefficient variation as a function of wavelength in the case of a sample consisting of gold sulfide coated gold nanorods. Three main peaks can be seen as indicated by 1, 2 and 3 in the figure. The peaks 1 and 2 were explained as arising due to longitudinal and transverse modes of plasmon resonance, respectively of the gold nanorods. This was concluded by fitting the experimental data to the equation for extinction coefficient deduced by Gans (1915) on the basis of extended Mie theory. The peak number 3 was analysed on the basis of a core-shell structure of the rods (Zhou et al 1994). The small hump in the extinction coefficient described by 4 in figure 8 was ascribed to the presence of gold nanoparticles. It should be mentioned here that the gold nanorods as observed by transmission electron microscopy comprised of interconnection between numerous gold particles. The details of analysis have been published elsewhere (Chatterjee et al 2006).

Table 1. Comparison of $d_{h k l}$ values obtained from electron diffraction with standard ASTM data for sulfide-coated gold nanorod.

\begin{tabular}{lcl}
\hline & \multicolumn{2}{c}{ ASTM } \\
\cline { 2 - 3 } Observed $d_{h k l}(\mathrm{~nm})$ & $\mathrm{Au}(\mathrm{nm})$ & $\mathrm{Au}_{2} \mathrm{~S}(\mathrm{~nm})$ \\
\hline 0.298 & & $0.289(111)$ \\
0.235 & $0.2355(111)$ & \\
0.201 & $0.2039(200)$ & $0.178(220)$ \\
$0 \cdot 178$ & $0.1442(220)$ & \\
$0 \cdot 143$ & & \\
\hline
\end{tabular}




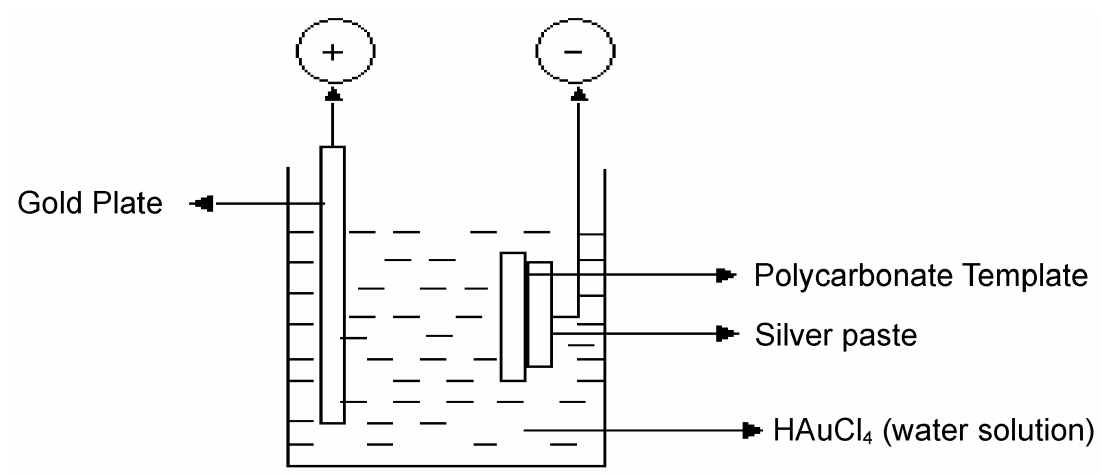

Figure 6. Experimental arrangement for growing gold nanowires.

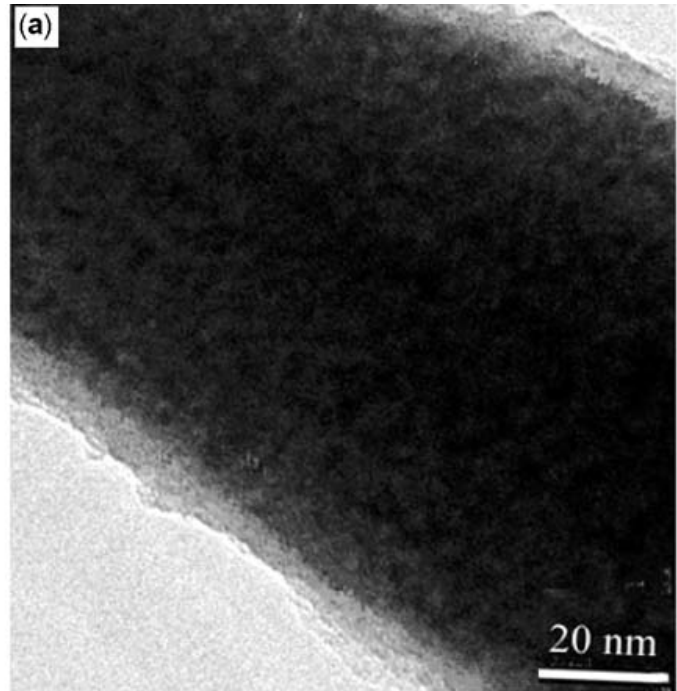

\section{(b)}

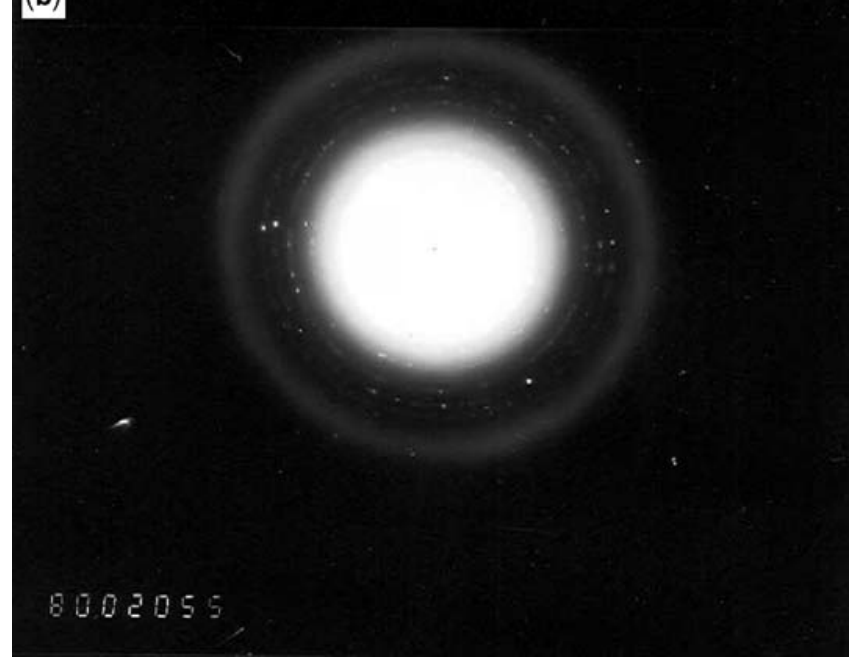

Figure 7. (a) Transmission electron micrograph for gold sulfide coated gold nanorod and (b) electron diffraction pattern obtained from figure $7(a)$

\subsection{Silver oxide and silver nanoparticles in polymer film}

Figure 9 is a transmission electron micrograph of $\mathrm{Ag}_{2} \mathrm{O}$ particles prepared here. The electron diffraction pattern

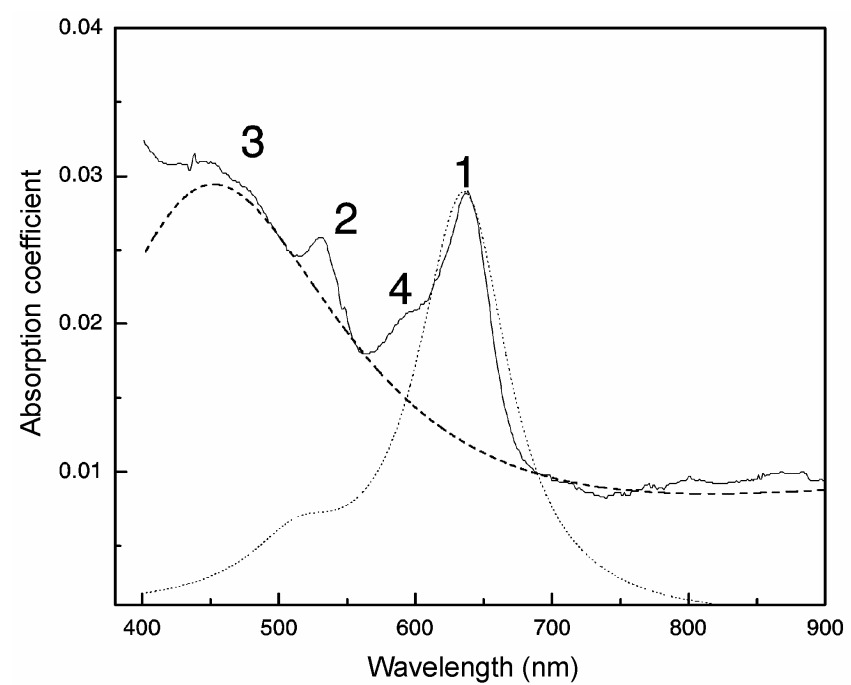

Figure 8. Optical absorption spectrum for gold sulfide coated gold nanorods as a function of wavelength at $300 \mathrm{~K}$.

confirmed the particles to be made up of $\mathrm{Ag}_{2} \mathrm{O}$. Silver nanoparticles distribution was similar. On analysing the particle sizes by a log normal distribution function, the extracted values of median diameter and geometric standard deviation were found to be $8.5 \mathrm{~nm}$ and 1.2 , respectively.

An a.c. field of $10 \mathrm{~V} / \mathrm{mm}$ at a frequency of $50 \mathrm{~Hz}$ formed a string like structure parallel to the electrodes. Such a pattern formation is explained as arising due to electrohydrodynamic (EDH) fluid flow (Trau et al 1997).

Figure 10 shows the scanning electron micrograph for the binary nanoparticles under the influence of an a.c. field of $10 \mathrm{~V} / \mathrm{mm}$ at a frequency of $1 \mathrm{MHz}$. The alignment of the particle arrays perpendicular to the electrodes has been explained on the basis of interaction energy between the dipoles associated with the nanoparticles. Using the expression for dipole-dipole interaction and the polarizabilites of particles it was found that the interaction energies $U_{\mathrm{Ag}-\mathrm{Ag}}$ and $U_{\mathrm{Ag}_{\mathrm{Ag}} \mathrm{O}}$ were both negative but $U_{\mathrm{Ag}-\mathrm{Ag}}$ had a higher magnitude than $U_{\mathrm{Ag}_{-} \mathrm{Ag}_{2} \mathrm{O}}$ (Pal et al 2005). This explained the alignment of the particle arrays along the electric field direction. 
Figure 11 gives the resistivity variation as a function of inverse temperature for a PMMA film containing the aligned binary particles. The higher temperature region shows resistivity variation controlled by the statistically distributed silver nanoparticle pairs between which electron hopping takes place-the silver particles forming localized states.

Figure 12 gives the variation of resistivity of the PMMA film containing aligned binary nanoparticles as a function of relative humidity - the frequency of the a.c. field being $100 \mathrm{KHz}$ at which the alignment was brought about. It can be seen that the present system shows marked dependence of resistivity on relative humidity. This is

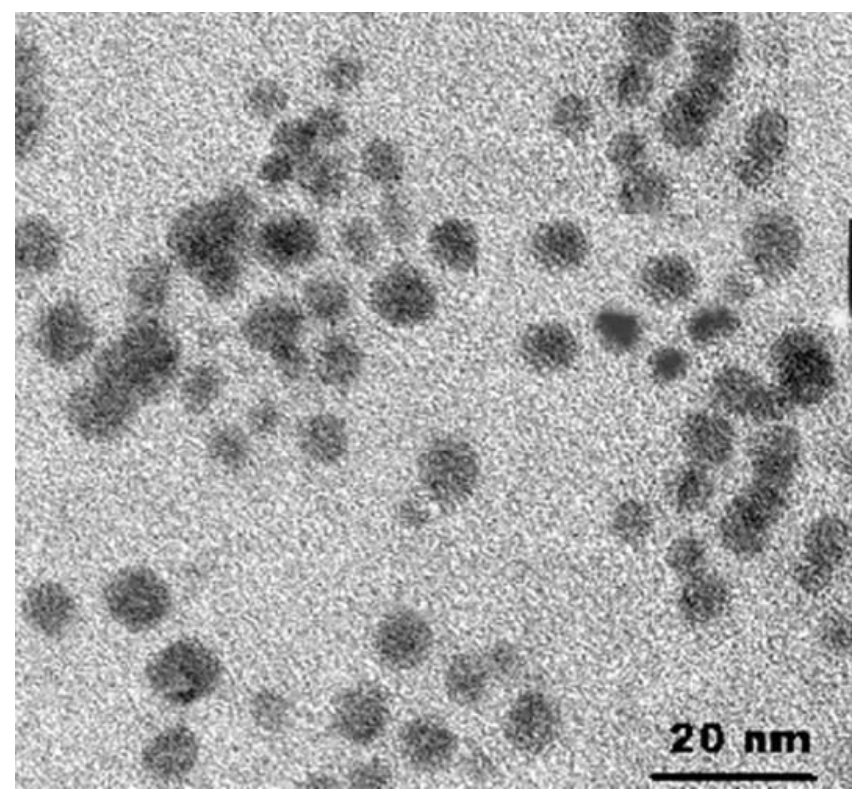

Figure 9. Transmission electron micrograph of $\mathrm{Ag}_{2} \mathrm{O}$ particles.

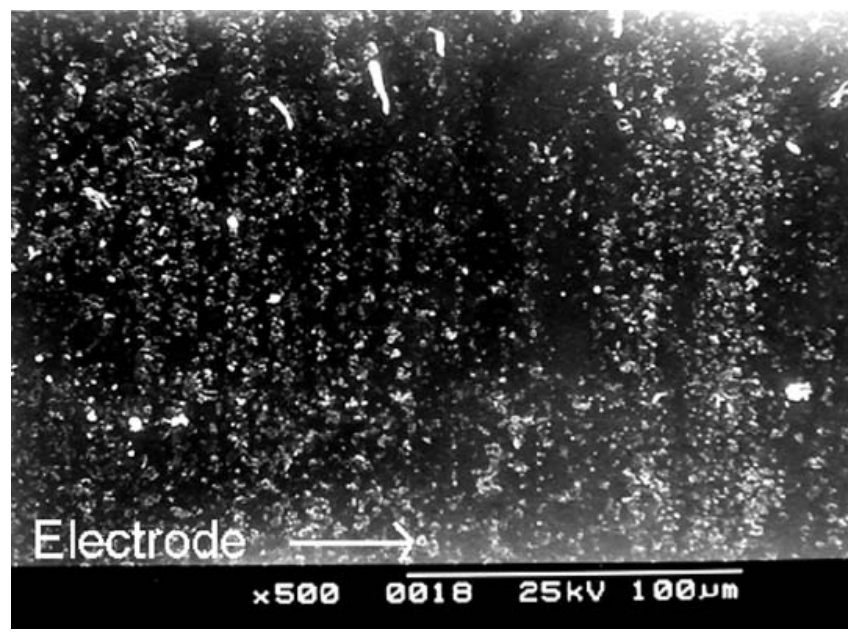

Figure 10. Scanning electron micrograph for the binary nanoparticles, $\mathrm{Ag}$ and $\mathrm{Ag}_{2} \mathrm{O}$. ascribed to an increase in the density of states resulting from the absorption of water molecules on the silver nanoparticles (Pal et al 2003).

\subsection{Lead sulfide nanowires in mica channels}

Figure 13(a) gives a dark field image of the transmission electron micrograph of $\mathrm{PbS}$ nanowires. In this figure the bright lines indicate the presence of nanowires. Figure 13(b) is the electron diffraction pattern obtained from

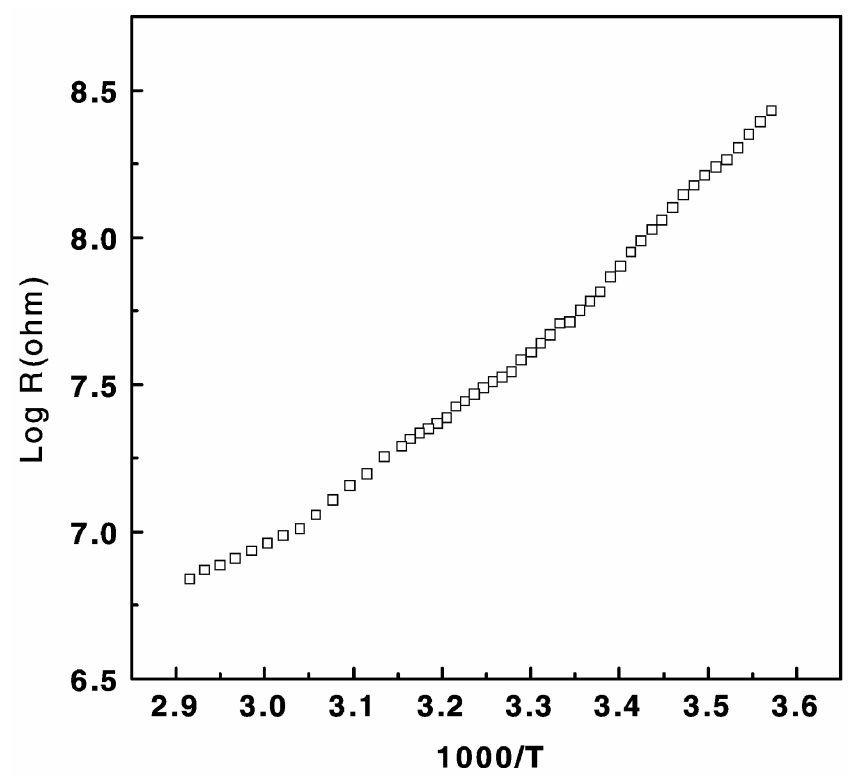

Figure 11. Variation of logarithm of resistance as a function of inverse temperature for PMMA film containing aligned binary nanoparticles of $\mathrm{Ag}$ and $\mathrm{Ag}_{2} \mathrm{O}$.

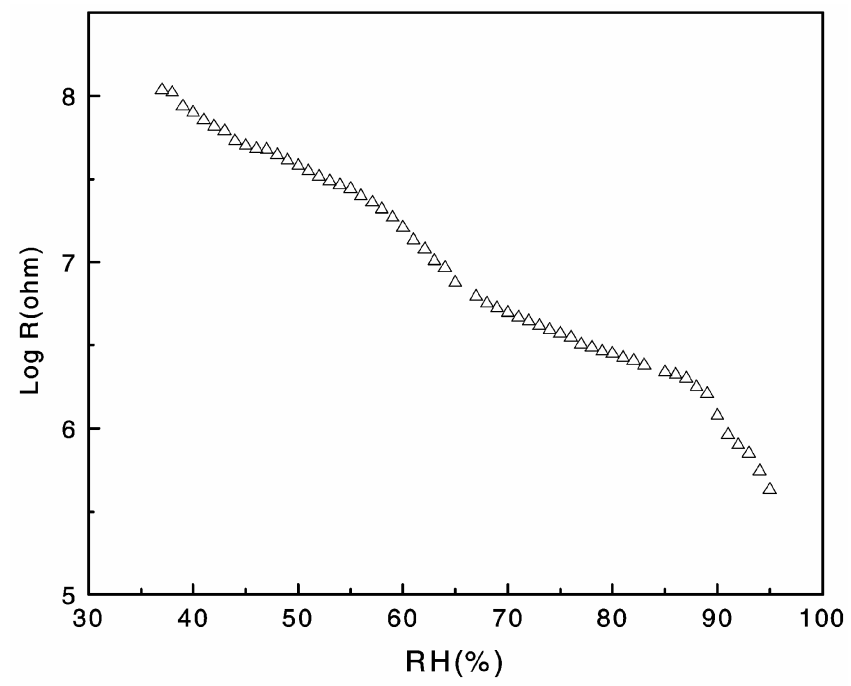

Figure 12. Variation of logarithm of resistance as a function of relative humidity for PMMA-binary nanoparticle composite aligned by a.c. field at $100 \mathrm{kHz}$. 
figure 13(a). The interplanar spacings calculated from the diffraction spots confirm the presence of $\mathrm{PbS}$ in the nanowires. From figure 13(a) it should be evident that many of the nanowires have break-gaps.

Figure 14 shows the variation of resistance of a nanocomposite of $\mathrm{PbS}$ in $\mathrm{Na}-4$ mica as a function of temperature. It can be seen that there is a transition from a semiconducting state to a metallic state at around $300 \mathrm{~K}$. The bandgap calculated from the slope of $\log R$ vs $1 / T$ was found to be $0.32 \mathrm{eV}$ which is consistent with the semi-
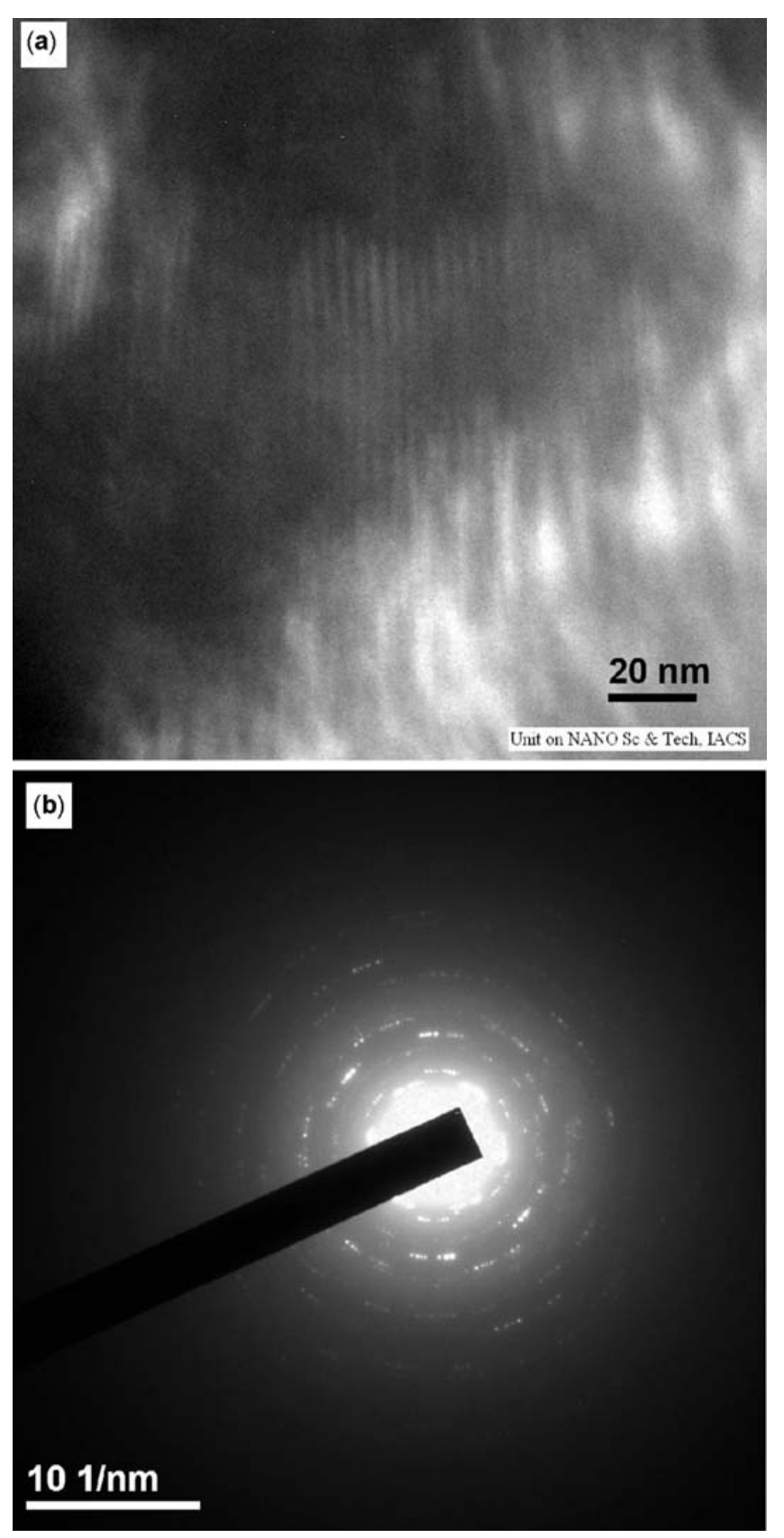

Figure 13. (a) Dark field image of transmission electron micrograph for $\mathrm{PbS}$ nano wires grown within $\mathrm{Na}-4$ mica channels and (b) electron diffraction pattern obtained from figure 13(a). conducting phase, $\mathrm{PbS}$. The transition to a metallic state at temperatures below $300 \mathrm{~K}$ has been ascribed to the effect of high pressure generated by the mismatch of thermal expansion coefficients of mica and $\mathrm{PbS}$ phases. A pressure of $\sim 3.1 \mathrm{GPa}$ was calculated due to the cooling of the sample from $673 \mathrm{~K}$ (synthesis temperature) to $300 \mathrm{~K}$. The resistance change in the present system is, therefore, a direct consequence of a decrease in the bandgap with increasing pressure.

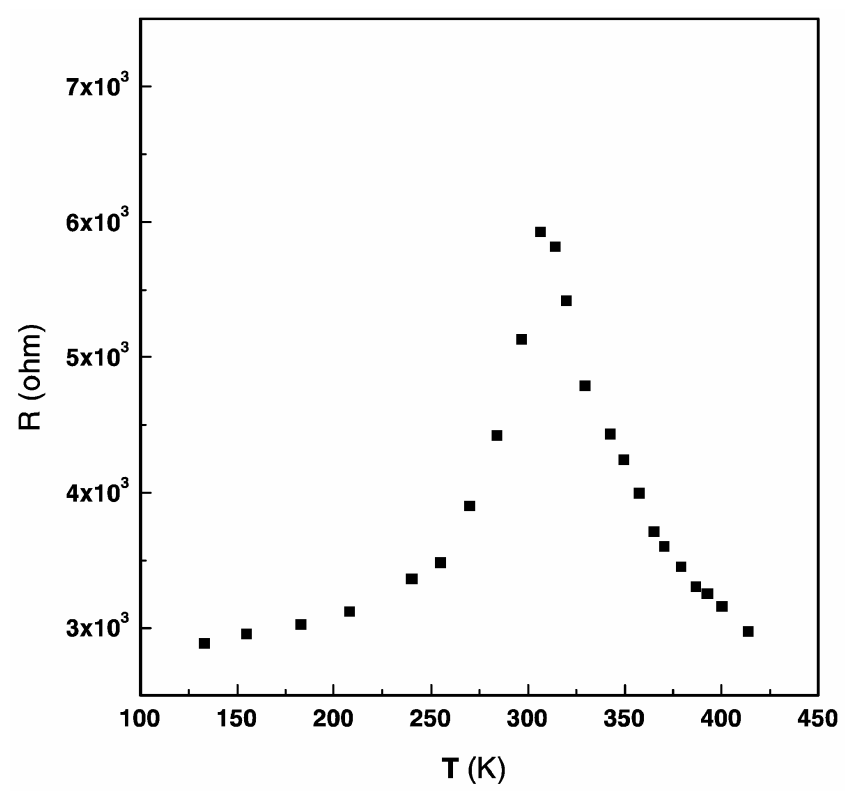

Figure 14. Resistance variation as a function of temperature for $\mathrm{PbS} / \mathrm{Na}-4$ mica nanocomposites.

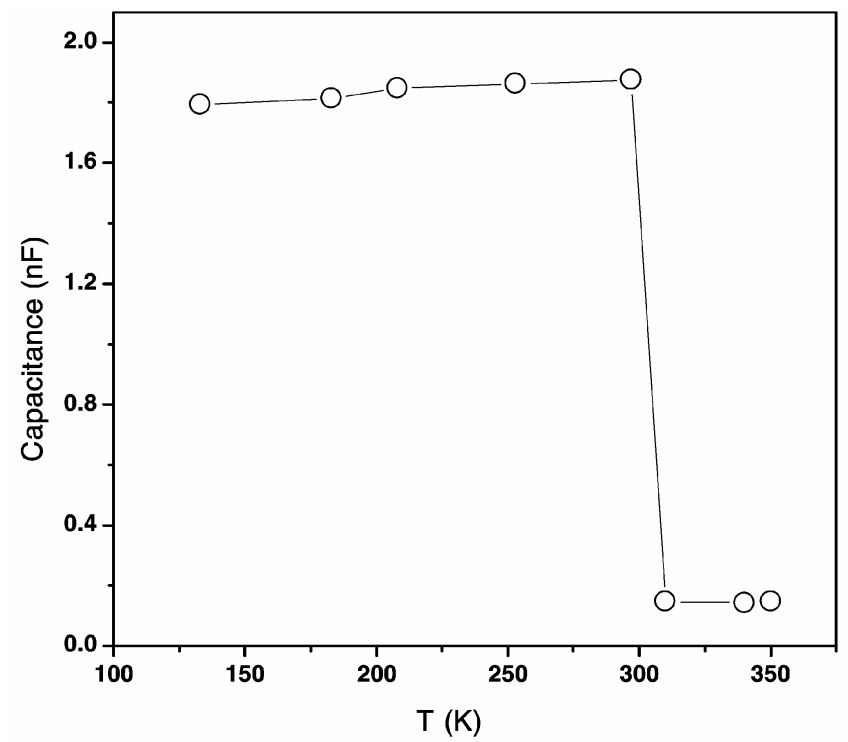

Figure 15. Variation of capacitance as a function of temperature for $\mathrm{PbS} / \mathrm{Na}-4$ mica nanocomposite. 
The semiconductor-metal transition is further corroborated by the drastic increase in the capacitance value of the sample as the temperature is lowered below $300 \mathrm{~K}$. Figure 15 depicts the variation of capacitance as a function of temperature for a nanocomposite comprising of $\mathrm{Na}-4$ mica and $\mathrm{PbS}$ nanowires. It has been shown earlier that interrupted strands of metal should have high dielectric permittivity (Rice and Bernasconi 1972). In the present specimen system there are broken $\mathrm{PbS}$ nanowires of average length, $\sim 20 \mathrm{~nm}$ (as estimated from electron micrographs). After they have undergone a transition to a metallic phase the predicted value of dielectric constant in accordance with Rice and Bernasconi (1972) model would be around 570. This is of the same order of magnitude as that calculated from the data in figure 15. Further details of the above analysis have been reported elsewhere (Mukherjee et al 2006a).

\subsection{Copper sulfide nanowires in Na-4 mica channels}

Figure 16 is a transmission electron micrograph for nanocomposite of Na-4 and copper sulfide nanowires. The diameters are seen to be $\sim 1.2 \mathrm{~nm}$. From electron diffraction spots we found that both $\mathrm{CuS}$ and $\mathrm{Cu}_{2} \mathrm{~S}$ were present in our system. Also there are segments of these nanowires present within the channels.

Figure 17 shows the variation of $\log R$ as a function of inverse temperature. The activation energy can be seen to be very small indicating transport by electron tunneling. It was reported earlier that $\mathrm{CuS}$ could exhibit metallic conduction (Gopalakrishnan et al 1979). We therefore conclude that $\mathrm{CuS}$ segments of diameter $17.4 \mathrm{~nm}$ (as deducted from X-ray line broadening of this phase) contribute to conduction by electron tunneling.

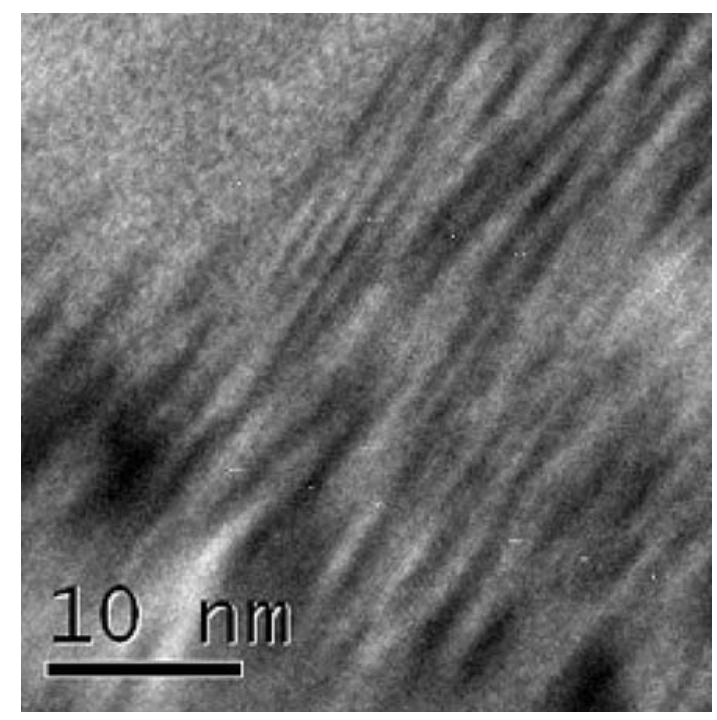

Figure 16. Transmission electron micrograph for a nanocomposite of Na-4 mica and copper sulfide nanowires.
Figure 18 shows the variation of capacitance of the Na-4 mica-copper sulfide nanocomposite as a function of frequency. The large value of dielectric permittivity, viz. 1617 as extracted from this result at high frequencies could be explained as arising due to Rice-Bernasconi mechanism explained earlier. The model predicted a value of 1682 in satisfactory agreement with the observed result.

The details of the above analysis have been discussed elsewhere (Mukherjee et al 2006b).

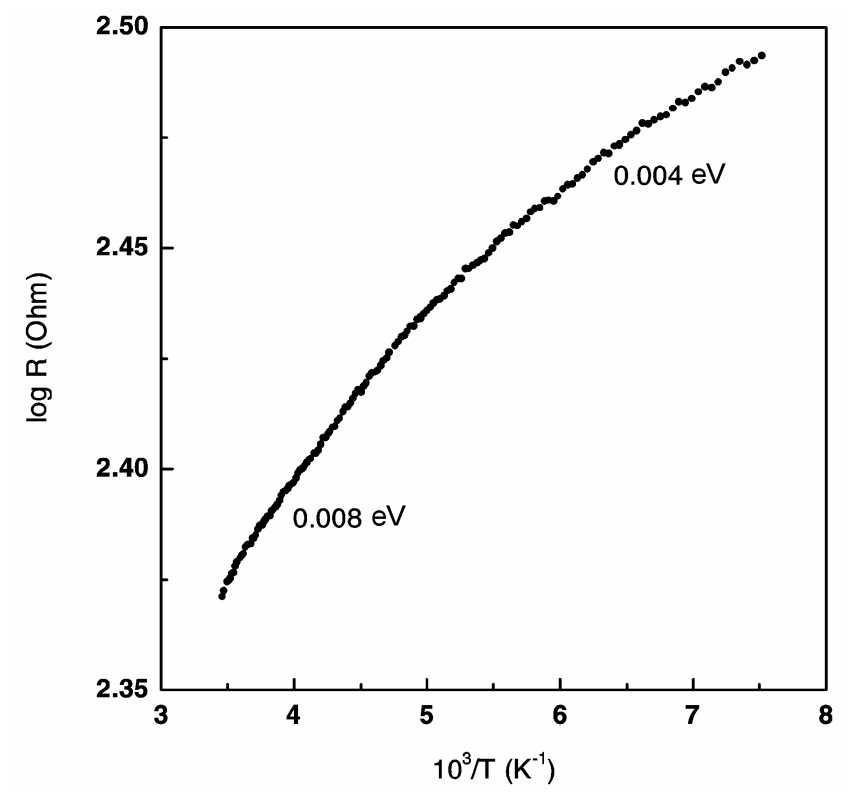

Figure 17. Variation of logarithm of resistance as a function of inverse temperature for a $\mathrm{Na}-4$ mica-copper sulfide wire nanocomposite.

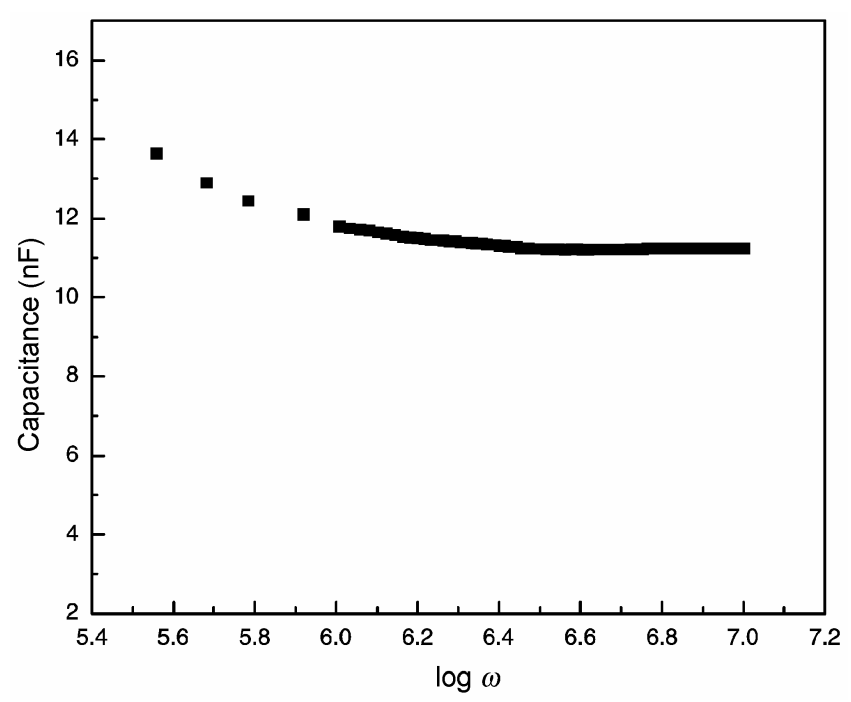

Figure 18. Variation of capacitance as a function of frequency for a Na-4 mica-copper sulfide wire nanocomposite. 


\subsection{Silver core-silver orthosilicate shell nanostructures}

Figure 19 is a typical transmission electron micrograph for a glass containing silver nanoparticles and subjected to a heat treatment at $833 \mathrm{~K}$ for $1 \mathrm{~h}$ followed by $993 \mathrm{~K}$ for $2 \mathrm{~h}$. From the figure it can be seen that the silver core has a diameter of $\sim 20 \mathrm{~nm}$ and the orthosilicate shell a thickness of $\sim 21 \mathrm{~nm}$. The electron diffraction pattern confirms the presence of silver orthosilicate shell.

Figure 20 shows the variation of logarithm of d.c. resistance as a function of inverse temperature for a glass sample containing silver-silver orthosilicate core-shell nanostructure. It can be seen from this figure that at tem-

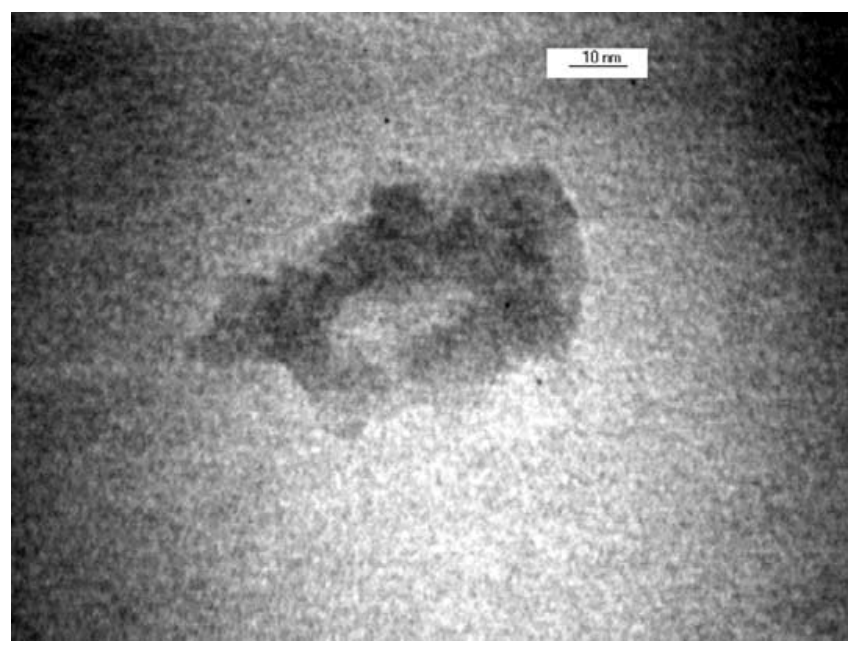

Figure 19. Transmission electron micrograph for a glass containing core-shell structure of silver nanoparticles and silver orthosilicate layers.

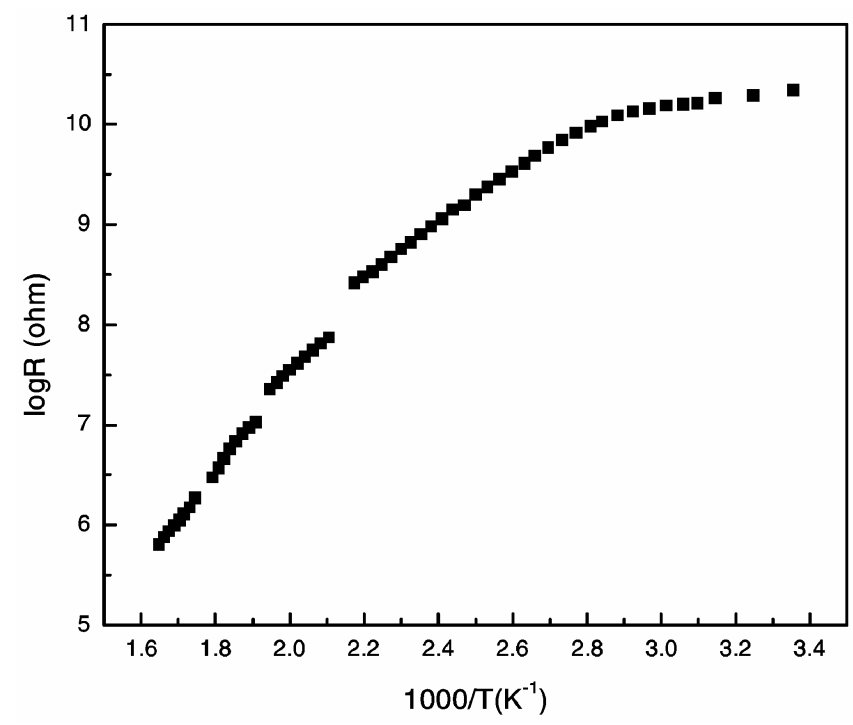

Figure 20. Variation of logarithm of d.c. resistance as a function of inverse temperature for a glass containing silver-silver orthosilicate core-shell nanostructure. peratures around $460 \mathrm{~K}, 510 \mathrm{~K}$ and $570 \mathrm{~K}$, the resistance shows discontinuous changes. This behaviour has been explained on the basis of high pressure generated on the shell phase due to thermal expansion mismatch between silver core and oxide shell. This causes a deformation in the shell perimeter and whenever it reaches a value equal to $\mathrm{Si}-\mathrm{O}$ distance (viz. $0.16 \mathrm{~nm}$ ), sharp change in resistance occurs. Considering the expression for electrical conductivity for an ionically conducting material, the preexponential factor contains the term, $v$, which is the ionic vibrational frequency. According to Lamb's (1882) theory, the spheroidal acoustic modes are quantized in terms of their frequencies. The present data were analysed on the basis of this theory and it was shown that the discontinuous changes in conductivity were a direct consequence of the excitation of Lamb modes (Ghosh et al 2004).

\subsection{Iron-iron oxide core-shell nanostructure and optical properties}

Figure 21 is a transmission electron micrograph showing the core-shell structure of a nanocomposite which was given an oxidation treatment at $573 \mathrm{~K}$ for $30 \mathrm{~min}$. The histogram of particle sizes showed a median diameter of $5 \cdot 3 \mathrm{~nm}$. From electron diffraction pattern of the core-shell structure it was concluded that both $\alpha-\mathrm{Fe}$ and $\mathrm{Fe}_{3} \mathrm{O}_{4}$ were present.

Figure 22 shows the variation of optical absorption as a function of wavelength for the nanocomposite which was given a heat treatment at $873 \mathrm{~K}$ for $30 \mathrm{~min}$. It is evident that there are three absorption peaks in the figure. The first one arises due to the presence of uncoated iron nanoparti-

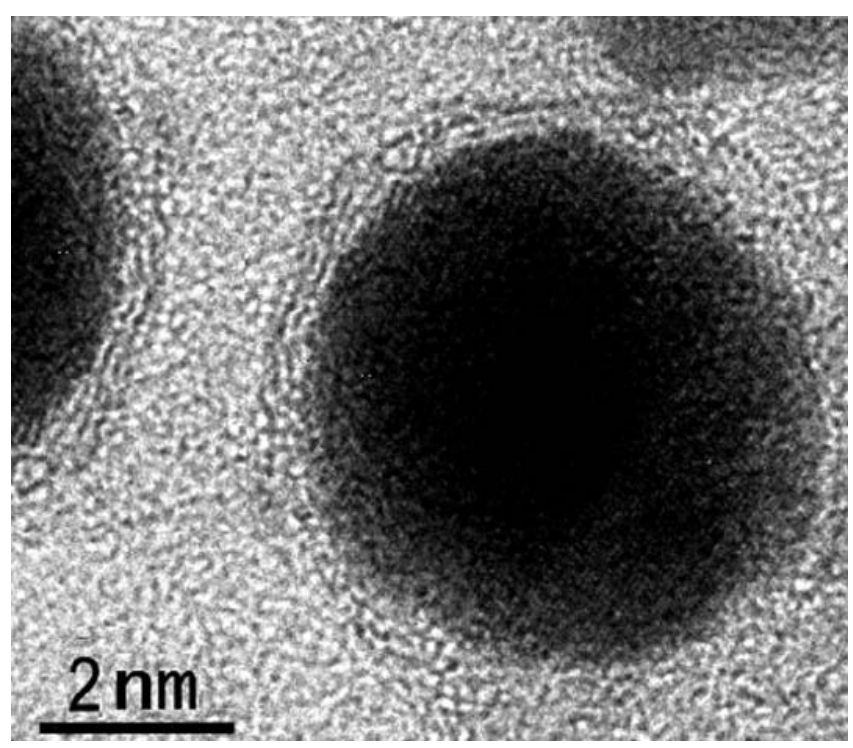

Figure 21. Transmission electron micrograph of an iron coreiron oxide shell nanostructure in a glass medium 
cles and is analysed by applying Maxwell-Garnet model (Maxwell-Garnet 1904) according to which the effective permittivity of the medium, $\bar{\varepsilon}$, can be written as

$$
\bar{\varepsilon}=\frac{2 f \varepsilon_{\mathrm{m}} \varepsilon_{\mathrm{h}}-2 f \varepsilon_{\mathrm{h}}^{2}+\varepsilon_{\mathrm{m}} \varepsilon_{\mathrm{h}}+2 \varepsilon_{\mathrm{h}}^{2}}{\varepsilon_{\mathrm{m}}+2 \varepsilon_{\mathrm{h}}-f \varepsilon_{\mathrm{m}}+f \varepsilon_{\mathrm{h}}},
$$

where $f$ is the volume fraction of the metallic phase, $\varepsilon_{\mathrm{m}}$ the dielectric permittivity of the latter and $\varepsilon_{\mathrm{h}}$ the dielectric permittivity of the host medium (in our case silicate glass). The expression for $\varepsilon_{\mathrm{m}}$ is as given below (Kreibig 1974)

$$
\varepsilon_{\mathrm{m}}=1-\frac{\omega_{\mathrm{p}}^{2}}{\omega^{2}-i \omega \sigma / \varepsilon_{0}},
$$

where $\omega_{\mathrm{p}}$ is the plasmon frequency for iron, $\sigma$ the conductivity of iron particle and $\varepsilon_{0}$ the free space permittivity. The calculated value of plasmon frequency of iron is $2.3 \times 10^{16} \mathrm{rad} / \mathrm{s}$ (Kittel 1961). The optical absorption coefficient, $\mu$, is given by (Chatterjee et al 1989)

$$
\mu=\frac{\omega}{c} \frac{\bar{\varepsilon}_{2}}{\sqrt{\bar{\varepsilon}_{1}}},
$$

where $\omega$ is the angular frequency, $c$ the velocity of light, $\bar{\varepsilon}_{1}$ and $\bar{\varepsilon}_{2}$ are the real and imaginary parts, respectively of $\bar{\varepsilon}$.

The first peak was fitted to (2), (3) and (4) taking $\sigma, f$ and $\varepsilon_{\mathrm{h}}$ as parameters. In the figure the dotted line represents the theoretical fit and the solid line corresponds to experimental data. The values extracted for the parameters were $\sigma=3.9 \times 10^{4}(\mathrm{ohm} \mathrm{m})^{-1}, f=1 \times 10^{-3}$ and $\varepsilon_{\mathrm{h}}=3.8$. These values are reasonable for our system.

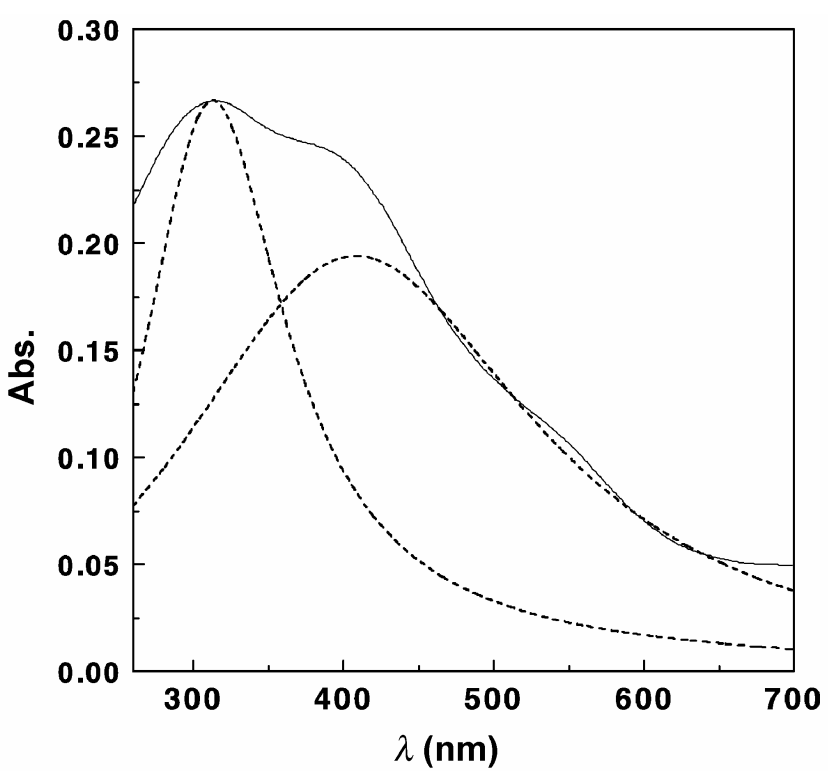

Figure 22. Optical absorption as a function of wavelength for a nanocomposite with iron core-iron oxide shell nanostructure.
The second peak was analysed on the basis of the presence of core-shell structured nanoparticles. The polarizability, $\alpha$, in this case can be written as (Zhou et al 1994)

$$
\alpha=\sum_{j} \frac{n_{j} r_{j}^{3}}{\sum n_{j} r_{j}^{3}}\left\{\begin{array}{l}
\left(\varepsilon_{\mathrm{s}}-\varepsilon_{\mathrm{h}}\right)\left(\varepsilon_{m j}+2 \varepsilon_{\mathrm{s}}\right)+\left(1-\frac{t_{j}}{r_{j}}\right)^{3} \\
\frac{\left(\varepsilon_{m j}-\varepsilon_{\mathrm{s}}\right)\left(\varepsilon_{\mathrm{h}}+2 \varepsilon_{\mathrm{s}}\right)}{\left(\varepsilon_{m j}+2 \varepsilon_{\mathrm{s}}\right)\left(2 \varepsilon_{\mathrm{h}}+\varepsilon_{\mathrm{s}}\right)+2\left(1-\frac{t_{j}}{r_{j}}\right)} \\
\left(\varepsilon_{\mathrm{s}}-\varepsilon_{\mathrm{h}}\right)\left(\varepsilon_{m j}-\varepsilon_{\mathrm{s}}\right)
\end{array}\right\} r_{j}^{3},
$$

where $\varepsilon_{\mathrm{s}}$ is the dielectric permittivity of the shell, $n_{j}$ the number of particles having radius $r_{j}$ and $t_{j}$ the thickness of the shell on the particle $r_{j}$. The size dependent dielectric permittivity, $\varepsilon_{m j}$, for a metal core with radius, $r_{j}$, is given by (Kreibig 1974)

$$
\varepsilon_{m j}=\left(1-\frac{\omega_{\mathrm{p}}^{2}}{\omega^{2}}\right)+i\left[\frac{\omega_{\mathrm{p}}^{2}}{\omega^{3}}\left(\frac{\sigma_{m j}}{\varepsilon_{\mathrm{o}}}+\frac{V_{\mathrm{F}}}{r_{j}-t_{j}}\right)\right],
$$

where $\sigma_{\mathrm{mj}}$ is the electrical conductivity of the metal core with radius $\left(r_{j}-t_{j}\right), \varepsilon_{\mathrm{o}}$ the free space permittivity and $V_{\mathrm{F}}$ the Fermi velocity for iron. Using Clausius-Mosotti relation (Kittel 1961), the dielectric permittivity of the composite system can be written as

$$
\alpha=\frac{1+2 N \alpha}{1-N \alpha}
$$

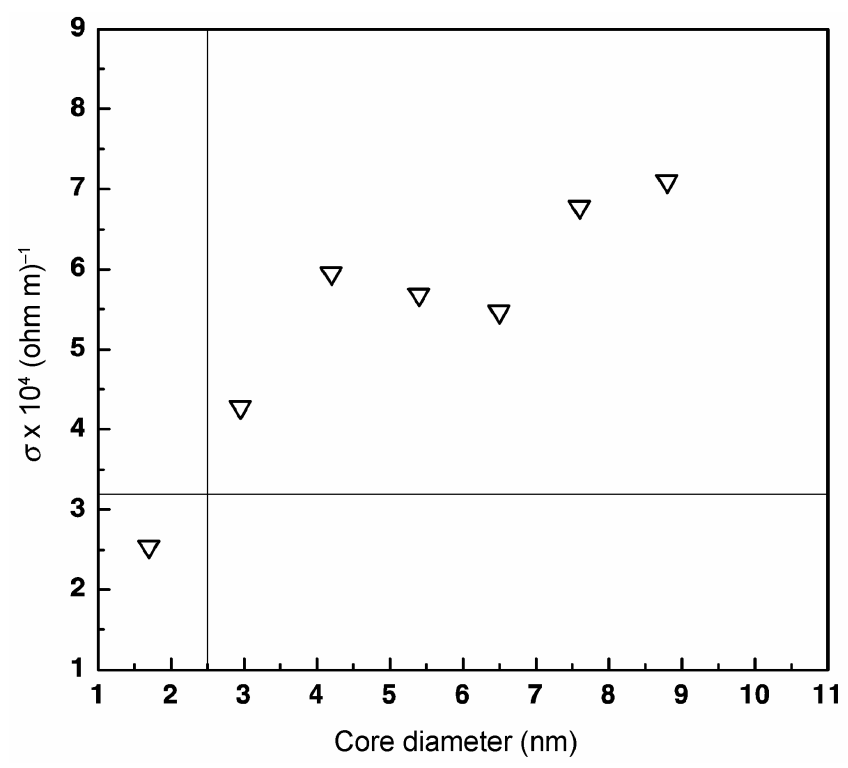

Figure 23. Extracted values of electrical conductivity as a function of iron particle diameter in the case of sample heat treated at $873 \mathrm{~K}$ for $30 \mathrm{~min}$ 
where $N$ is the number of nanoparticles per unit volume. Using (5), (6) and (7), the second peak was fitted using $N, \varepsilon_{\mathrm{s}}, t_{j}$ and $\sigma_{m j}$ as the variable parameters. The dotted line in figure 22 shows the theoretical fit. The values of $\sigma_{m j}$ as extracted from the fitting are summarized in figure 23 . It can be seen that for core diameter of metallic iron below
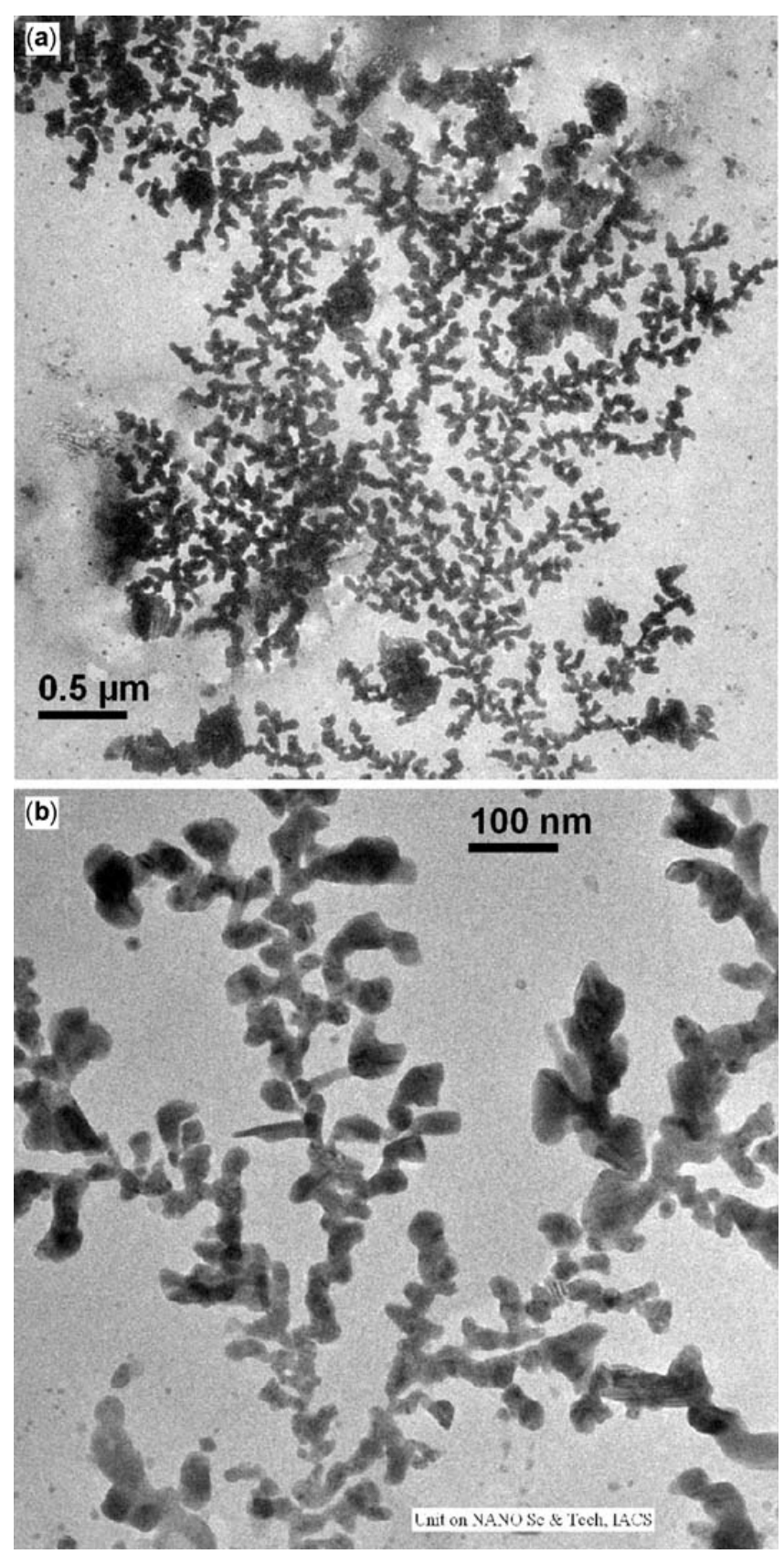

Figure 24. Transmission electron micrograph of dendron-like growth of silver particles using tetrapeptide at different magnifications: (a) scale bar, $0.5 \mu \mathrm{m}$ and (b) scale bar, $100 \mathrm{~nm}$. (Reprinted figure with permission from Santanu Bhattacharya, Apurba K Das, Arindam Banerjee and Dipankar Chakravorty 2006 J. Phys. Chem. B110 10758; Copyright @ American Chemical Society (2006)). $\sim 2.5 \mathrm{~nm}$ the conductivity values extracted by this fitting procedure fall below Mott's minimum metallic conductivity which is given by

$$
\sigma_{\text {min }} \cong\left(\frac{0 \cdot 06 e^{2}}{\hbar a}\right)\left(\frac{6}{z}\right)^{2},
$$
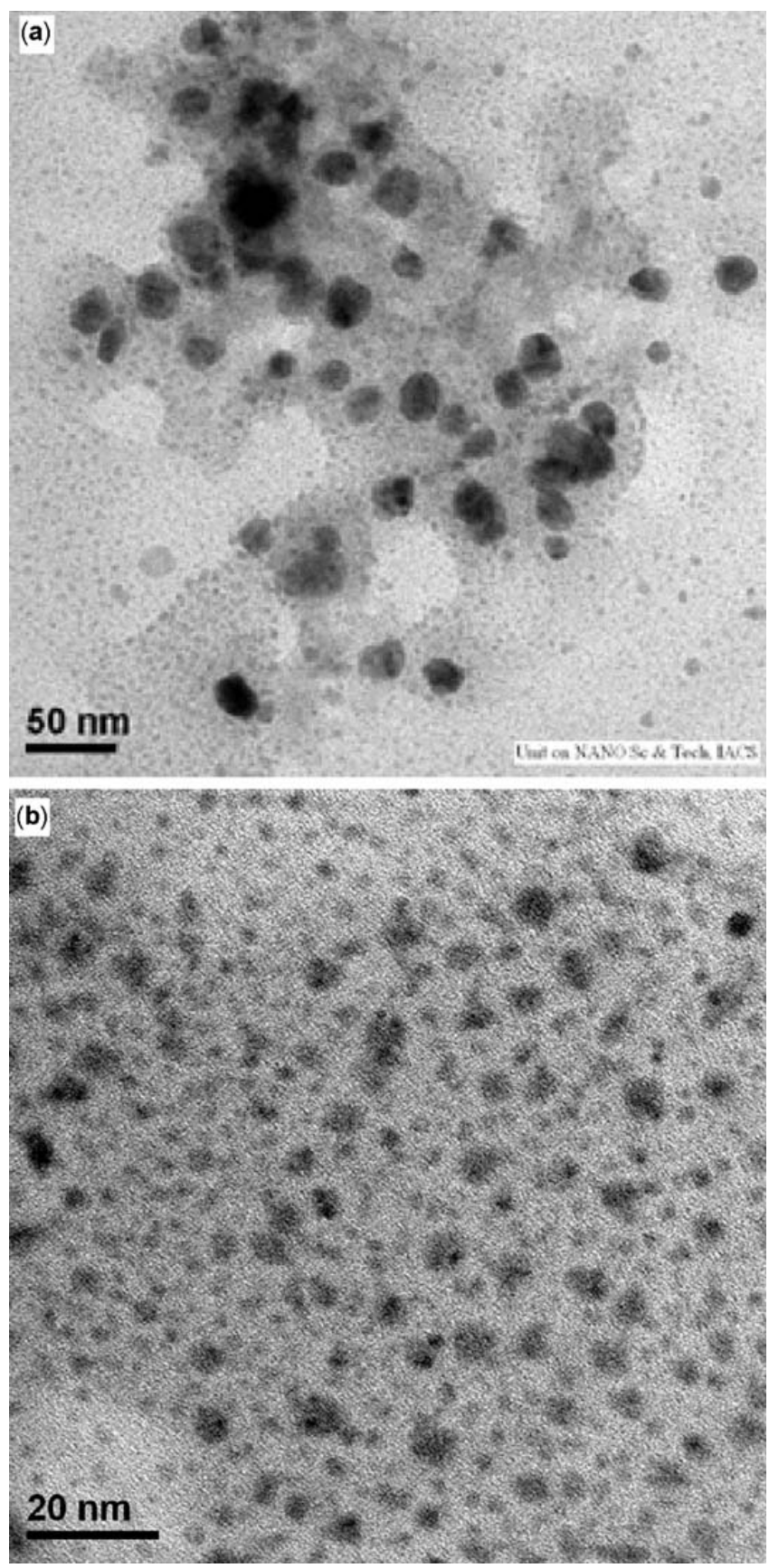

Figure 25. Nanoparticles of silver in the peptide solution: (a) scale bar, $50 \mathrm{~nm}$ and (b) scale bar, $20 \mathrm{~nm}$. (Reprinted figure with permission from Santanu Bhattacharya, Apurba K Das, Arindam Banerjee and Dipankar Chakravorty 2006 J. Phys. Chem. B110 10758; Copyright @ American Chemical Society (2006)). 


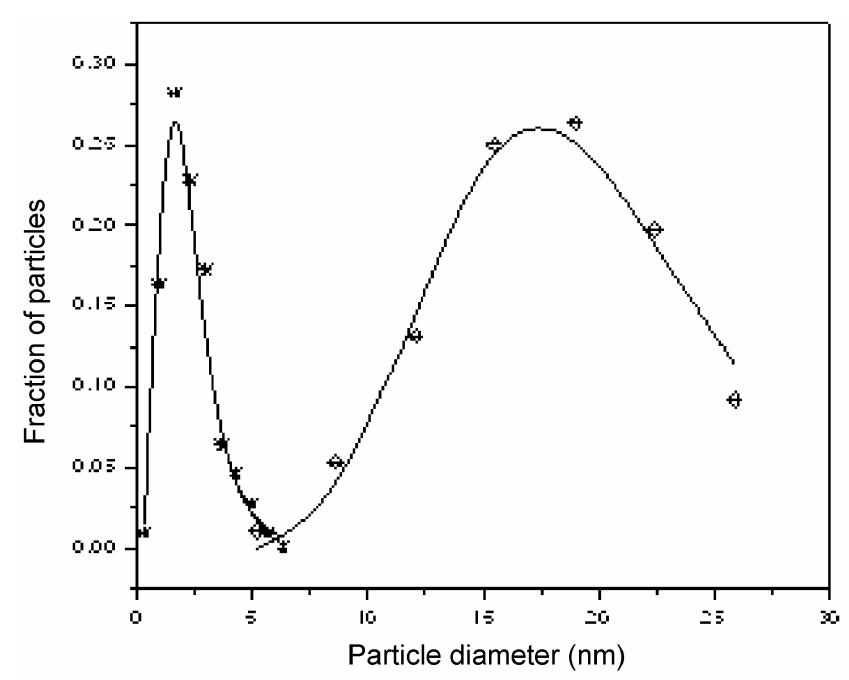

Figure 26. Particle size distribution obtained from figure 25 .

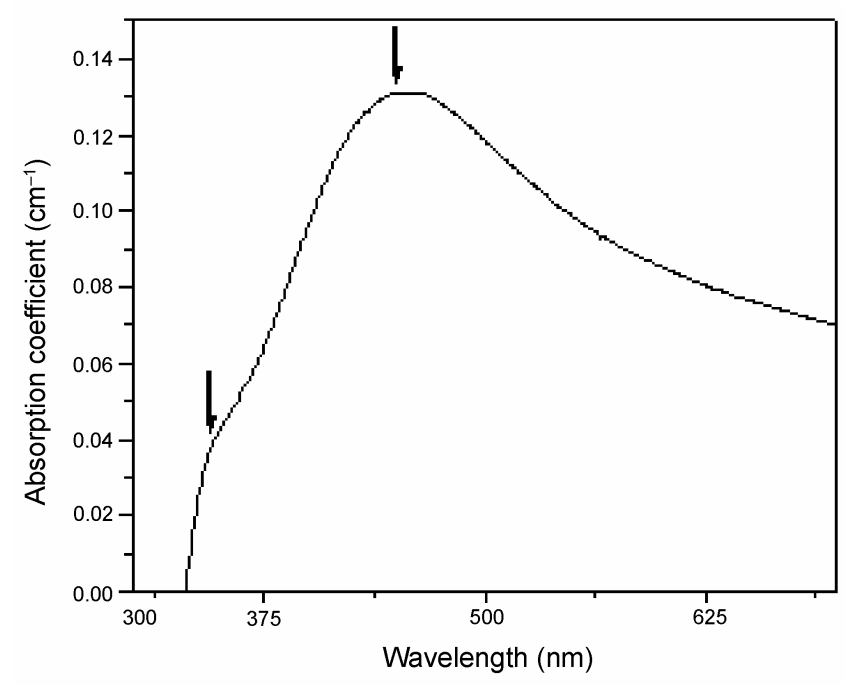

Figure 27. Optical absorption spectra for silver nanoparticles synthesized for a duration of $4 \mathrm{~h}$.

where $e$ is the electronic charge, $\hbar$ the Planck's constant, ' $a$ ' the interatomic distance and $z$ the coordination number. For the present system the calculated value of $\sigma_{\min }$ is $\sim 3 \cdot 2 \times 10^{4}(\Omega \mathrm{m})^{-1}$. The third peak at $550 \mathrm{~nm}$ is explained to arise due to $d-d$ transition (Jitianu et al 2002).

\subsection{Silver nanoparticles in oligopeptide}

Figures 24(a) and (b) are the transmission electron micrographs of the dendron-like structure formed by silver nanoparticles. Figures 25(a) and (b) are electron micrographs at a higher magnification showing the silver particle size distribution. In figure 26 is shown the particle size distribution. It is seen that a bi-modal distribution
Table 2. Summary of extracted values of electrical conductivity for metallic silver for different values of particle diameter.

\begin{tabular}{lc}
\hline Particle diameter $(\mathrm{nm})$ & Conductivity $\left(\Omega^{-1} \mathrm{~m}^{-1}\right)$ \\
\hline $0 \cdot 5$ & $0 \cdot 5$ \\
1 & $0 \cdot 7$ \\
$1 \cdot 5$ & $0 \cdot 8$ \\
2 & $1 \cdot 2$ \\
$2 \cdot 5$ & $1 \cdot 8$ \\
3 & $2 \cdot 4$ \\
$3 \cdot 5$ & $3 \cdot 0$ \\
4 & $3 \cdot 4$ \\
$4 \cdot 5$ & $3 \cdot 8$ \\
5 & $4 \cdot 3$ \\
$8 \cdot 6$ & $2 \cdot 3$ \\
12 & $2 \cdot 5$ \\
$15 \cdot 5$ & $2 \cdot 5$ \\
$18 \cdot 9$ & $2 \cdot 6$ \\
$22 \cdot 4$ & $2 \cdot 8$ \\
\hline
\end{tabular}

has been obtained, the mean diameters being $2 \mathrm{~nm}$ and $19.5 \mathrm{~nm}$, respectively. This is reflected in the optical absorption spectra shown in figure 27 where two peaks are observed-the peak at $340 \mathrm{~nm}$ is due to particles with a median diameter, $2 \mathrm{~nm}$ and the peak at $450 \mathrm{~nm}$ arises due to those with a median diameter, $19.5 \mathrm{~nm}$. This has been confirmed by analysing the absorption data on the basis of Mie scattering theory (Mie 1908) according to which the absorption coefficient, $\eta$, is given by

$$
\eta=\sum_{j} \frac{18 \pi f_{j} \varepsilon_{\mathrm{m}}^{3 / 2} \varepsilon_{2 j}}{\lambda\left[\left(\varepsilon_{1 j}+2 \varepsilon_{\mathrm{m}}\right)^{2}+\varepsilon_{2 j}^{2}\right]},
$$

where $f_{j}$ is the volume fraction of particles with diameter, $d_{j}, \varepsilon_{\mathrm{m}}$ the dielectric constant of the medium (methanol in our case) and $\varepsilon_{1 j}$ and $\varepsilon_{2 j}$ are the real and imaginary parts of the dielectric permittivity of the metal particle with diameter, $d_{j}$. The size dependent dielectric permittivity of a metal particle was calculated by (6). The experimental data of absorption coefficient were satisfactorily fitted to (9) and (6) using $\sigma_{j}, f$ and $\varepsilon_{\mathrm{m}}$ as parameters. The extracted values of conductivity for different particle diameters are summarized in table 2. It can be seen from these values that below a particle diameter of $2 \mathrm{~nm}$, the conductivities have values below Mott's minimum metallic conductivity which in the present case was calculated using (8) to be $1.2 \times 10^{4}(\mathrm{ohm})^{-1}$.

\section{Acknowledgements}

The work was supported by DST, New Delhi, under Nano Science and Technology Initiative Programme, S Basu, B N Pal, P K Mukherjee, B Ghosh, K Chatterjee, A Bose and S Bhattacharya thank CSIR, New Delhi, for awarding Senior Research Fellowships. (DC) thanks INSA, New 
Delhi, for the award of a Senior Scientist position. The authors thank Supriyo Chakraborty for providing skillful assistance in transmission electron microscopy.

\section{References}

Banerjee Sourish, Banerjee Soomit, Datta Anindya and Chakravorty Dipankar 1999 Europhys. Lett. 46346

Basu S and Chakravorty D 2006 J. Non-Cryst. Solids 352 380

Bhattacharya Santanu, Das Apurba K, Banerjee Arindam and Chakravorty Dipankar 2006 J. Phys. Chem. B110 10757

Bodanszky M and Bodanszky A 1984 The practice of peptide synthesis (New York: Springer-Verlag) pp 1-282

Chatterjee A and Chakravorty D 1989 J. Phys. D22 1386

Chatterjee K, Basu S and Chakravorty D 2006 J. Mater. Res. 21 34

Dutta P, Manivannan A, Seehra M S, Shah N and Huffman G P 2006 J. Appl. Phys. $9908 \mathrm{H} 105$

Gans R 1915 Ann. Phys. 47270

Ghosh B, Banerjee S, Ghosh A and Chakravorty D 2004 Phys. Rev. B70 195414

Gopalakrishnan J, Murugesan T, Hegde M S and Rao C N R 1979 J. Phys. C12 5255

Jitianu A, Crisan M, Megha A, Aru I and Zaharescu M $2002 \mathrm{~J}$. Mater. Chem. 121401

Kesanli B, Hong K, Meyer K, Im H-J and Dai S 2006 Appl. Phys. Lett. 89214104
Kittel C 1961 Introduction to solid state physics (New York: Wiley) p. 374

Kodama T and Komarneni S 1999 J. Mater. Chem. 9533

Kreibig U 1974 J. Phys. F: Met. Phys. 4999

Lamb H 1882 London Math. Soc. 13189

Matshushita M, Hond K, Toyoki H, Hayakawa Y and Kondo H 1986 J. Phys. Soc. Jpn 552618

Maxwell-Garnet J C 1904 Trans. R. Soc. London A203 385

Mie G 1908 Ann. Phys. 25377

Mott N F 1968 J. Non-Cryst. Solids 141

Mukherjee P K, Chatterjee K and Chakravorty D 2006a Phys. Rev. B73 035414

Mukherjee Pijush Kanti, Bose Anindita and Chakravorty Dipankar 2006 Appl. Phys. Lett. 89033101

$\mathrm{Ng} \mathrm{C} \mathrm{Y,} \mathrm{Chen} \mathrm{T} \mathrm{P,} \mathrm{Ding} \mathrm{L,} \mathrm{Liu} \mathrm{Y,} \mathrm{Tse} \mathrm{M} \mathrm{S,} \mathrm{Fung} \mathrm{S} \mathrm{and} \mathrm{Dong}$ Z L 2006 Appl. Phys. Lett. 88063103

Pal B N, Kundu T K, Banerjee S and Chakravorty D $2003 \mathrm{~J}$. Appl. Phys. 934201

Pal B N, Basu S and Chakravorty D 2005a J. Appl. Phys. 97 034311

Pal B N, Basu S and Chakravorty D 2005b J. Appl. Phys. 98 084306

Park J H and Park O O 2006 Appl. Phys. Lett. 89193101

Rice M J and Bernasconi J 1972 Phys. Rev. Lett. 29113

Trau M, Saville A and Aksay I A 1997 Langmuir 136375

Watt A, Eichmann T, Rubinsztein-Dunlop H and Meredith P 2005 Appl. Phys. Lett. 87253109

Zhou H S, Honma I, Komiyama H and Haus J W 1994 Phys. Rev. B50 12052 